\title{
THE ORTHOGONAL SUBCATEGORY PROBLEM AND THE SMALL OBJECT ARGUMENT
}

\author{
JIŘÍ ADÁMEK, MICHEL HÉBERT AND LURDES SOUSA
}

\begin{abstract}
A classical result of P. Freyd and M. Kelly states that in "good" categories, the Orthogonal Subcategory Problem has a positive solution for all classes $\mathcal{H}$ of morphisms whose members are, except possibly for a subset, epimorphisms. We prove that under the same assumptions on the base category and on $\mathcal{H}$, the generalization of the Small Object Argument of D. Quillen holds - that is, every object of the category has a cellular $\mathcal{H}$-injective weak reflection. In locally presentable categories, we prove a sharper result: a class of morphisms is called quasi-presentable if for some cardinal $\lambda$ every member of the class is either $\lambda$-presentable or an epimorphism. Both the Orthogonal Subcategory Problem and the Small Object Argument are valid for quasi-presentable classes. Surprisingly, in locally ranked categories (used previously to generalize Quillen's result), this is no longer true: we present a class $\mathcal{H}$ of morphisms, all but one being epimorphisms, such that the orthogonality subcategory $\mathcal{H}^{\perp}$ is not reflective and the injectivity subcategory $\operatorname{Inj} \mathcal{H}$ is not weakly reflective. We also prove that in locally presentable categories, the Injectivity Logic and the Orthogonality Logic are complete for all quasi-presentable classes.
\end{abstract}

\section{Introduction}

Our paper is devoted to two "classical" Galois connections between objects $A$ and morphisms $h$ of a given category $\mathcal{A}$ : orthogonality

$$
A \perp h,
$$

which means that the hom functor $\mathcal{A}[-, A]: \mathcal{A}^{o p} \rightarrow$ Set takes $h$ to an isomorphism, and injectivity

$$
A \in \operatorname{Inj}\{h\}
$$

which means that $\mathcal{A}[-, A]$ takes $h$ to an epimorphism.

(I) ORTHOGONALITY. Given a class $\mathcal{H}$ of morphisms in $\mathcal{A}$, the full subcategory

$$
\mathcal{H}^{\perp}
$$

Received December 17, 2007.

Financial support by Centre for Mathematics of University of Coimbra and by School of Technology of Viseu is acknowledged by the third author. 
of all objects orthogonal to all the members of $\mathcal{H}$ is clearly closed under limits. In [9], Peter Freyd and Max Kelly posed the Orthogonal Subcategory Problem:

is $\mathcal{H}^{\perp}$ a reflective subcategory of $\mathcal{A}$ ?

They proved that in "suitable" categories, the answer is affirmative whenever $\mathcal{H}$ is the union of a set of morphisms and of a class of epimorphisms:

$$
\mathcal{H}^{\perp} \text { reflective if } \mathcal{H}=\mathcal{H}_{0} \cup \mathcal{H}_{e} \text { with } \mathcal{H}_{0} \text { small and } \mathcal{H}_{e} \subseteq \text { Epi. }
$$

We call such classes $\mathcal{H}$ quasi-small. The "suitability" conditions considered in [9] included the existence of limits; later Kelly [15] showed that this condition is not needed. We call categories satisfying the weakened conditions locally bounded. For example, the category Top of topological spaces is locally bounded, but also, as we will show below, every locally presentable category of Peter Gabriel and Friedrich Ulmer [10].

It has been known that the result of Freyd and Kelly extends to more general classes of morphisms, assuming that the base category is locally presentable:

(i) Under the set-theoretical Vopěnka's Principle, $\mathcal{H}^{\perp}$ is reflective for all (!) classes $\mathcal{H}$, as proved by Jiří Rosický and the first author [5].

Without set-theoretical restrictions, we can also say something more general by using the concept of $\lambda$-presentable morphism as in [13]: it is a morphism $f: A \rightarrow B$ which, in the slice category $A \downarrow \mathcal{A}$, is a $\lambda$-presentable object. A class of morphisms is called presentable if there exists a cardinal number $\lambda$ such that all its members are $\lambda$-presentable. Every set of morphisms (in locally presentable categories) is presentable, but one easily finds presentable proper classes.

(ii) For all presentable classes $\mathcal{H}$, the subcategory $\mathcal{H}^{\perp}$ is reflective, see [2].

We are going to prove a joint generalization of the Freyd-Kelly result and of (ii), in the context of locally presentable categories:

$\mathcal{H}^{\perp}$ is reflective for all classes $\mathcal{H}=\mathcal{H}_{0} \cup \mathcal{H}_{e}$ with $\mathcal{H}_{0}$ presentable and $\mathcal{H}_{e} \subseteq$ Epi.

We call such classes $\mathcal{H}$ quasi-presentable. The results above concern the "object side" of the Galois correspondence $\perp$ between objects and morphisms. The "morphism side" is the field of Orthogonality Logic: given a class $\mathcal{H}$ of morphisms, characterize all its orthogonality consequences, that is, all morphisms $k$ such that all objects orthogonal to $\mathcal{H}$ are also orthogonal to $k$. 
Notation:

$$
\mathcal{H} \models k \text { iff } \mathcal{H}^{\perp} \subseteq\{k\}^{\perp}
$$

Inspired by the work of Grigori Roşu [21], we have recently formulated a simple deduction system for the Orthogonality Logic, and proved that it is sound and complete in every locally presentable category, for all classes $\mathcal{H}=\mathcal{H}_{0} \cup \mathcal{H}_{\text {se }}$, where $\mathcal{H}_{0}$ is small and the members of $\mathcal{H}_{\text {se }}$ are strong epimorphisms. Here we improve this result: the Orthogonality Logic is sound and complete for all quasi-presentable classes of morphisms. Not too surprisingly: assuming Vopěnka's Principle, soundness and completeness hold for all classes of morphisms. By applying the technique of Max Kelly, we also prove that in every locally bounded category, the soundness and completeness hold for all quasi-small classes of morphisms.

(II) INJECTIVITY. Here, the corresponding full subcategory

$$
\operatorname{Inj} \mathcal{H}
$$

of all objects injective with respect to all members of $\mathcal{H}$ is closed under products and split subobjects, but in general not under limits. Thus, we do not expect it to be reflective. However, in homotopy theory, weak reflections in subcategories of the form Inj $\mathcal{H}$ are often used. Recall that a weak reflection of an object $A$ in Inj $\mathcal{H}$ is a morphism $r_{A}: A \rightarrow A^{\prime}$ which is weakly initial in the slice category $A \downarrow \operatorname{Inj} \mathcal{H}$. Since weak reflections are not essentially unique, we may wish the morphism $r_{A}$ to have additional qualities. In homotopy theory, the requirement is that $r_{A}$ be $\mathcal{H}$-cellular, which means that it is a transfinite composite of morphisms, each of which is a pushout of a member of $\mathcal{H}$, see 3.2 below. A classical result of Daniel Quillen [20], called the Small Object Argument, states that if the object $A$ is "sufficiently small", then it has a cellular weak reflection in $\operatorname{Inj} \mathcal{H}$. A generalization is proved in [4]: for "sufficiently nice" categories, given any set $\mathcal{H}$ of morphisms, all objects have a cellular weak reflection in $\operatorname{Inj} \mathcal{H}$. In the present paper, we prove that the generalization of Quillen's Small Object Argument works:

(i) for quasi-small classes of morphisms in locally bounded categories, as well as

(ii) for quasi-presentable classes of morphisms in locally presentable categories.

Our proof of (i) is based on ideas of Max Kelly [15]. The categories used in [4] were quite similar to locally bounded ones, but did not allow the above 
generalization (from "small" to "quasi-small"): we demonstrate this in the last section by finding a category $\mathcal{A}$ satisfying the assumptions of [4], a class $\mathcal{H}$ whose members except one are epimorphisms and such that $\mathcal{H}^{\perp}$ is not reflective, and another such class with $\operatorname{Inj} \mathcal{H}$ not weakly reflective. In order to show this, we use substantially results from [18] and [19] on continuous algebras in $\mathrm{CPO}$.

There is, again, a "morphism side" to our Galois connection: the Injectivity Logic. Given a class $\mathcal{H}$ of morphisms, we want to characterize all its injectivity consequences, that is, all morphisms $k$ such that every object injective with respect to $\mathcal{H}$ is also injective with respect to $k$. Notation:

$$
\mathcal{H} \models k \text { iff } \operatorname{Inj} \mathcal{H} \subseteq \operatorname{Inj}\{k\}
$$

The Injectivity Logic has the same origin than the Orthogonality Logic, since Roşu ([21]) was dealing with classes of epimorphisms, in which case orthogonality and injectivity coincide. His logic was finitary (i.e., the deduction rules involved just a finite number of morphisms), and he proved the completeness for sets of finitely presentable epimorphisms with projective domains.

In the paper [8], the completeness was proved for finitary epimorphisms (i.e., with finitely presentable domains and codomains), considering a slight modification of Roçu's deduction system; moreover, it was shown that the completeness of the finitary logic may fail for sets of finitely presentable epimorphisms. In [1], this logic (and the corresponding completeness theorem) was extended to all finitary morphisms. Infinitary logic was also considered, and the completeness of the corresponding rules was proved for all classes of finitely presentable morphisms. Here we prove that the Injectivity Logic is sound and complete:

(i) for quasi-small classes of morphisms in locally bounded categories, as well as

(ii) for quasi-presentable classes of morphisms in locally presentable categories.

It is worth noting that, as opposed to the orthogonality case, no set-theoretical condition is needed to find a class $\mathcal{H}$ in a locally presentable category for which the Injectivity Logic is not complete: such an example is provided in [2] for the category of graphs.

Acknowledgement The authors are grateful to Jiří Rosický for interesting discussions about Theorem 2.6. 


\section{Locally Presentable and Locally Bounded Categories}

Throughout the paper we assume that the category $\mathcal{A}$ is cocomplete and $\mathcal{E}$ cowellpowered, where $(\mathcal{E}, \mathcal{M})$ is some fixed proper factorization system (i.e., $\mathcal{E} \subseteq$ Epi and $\mathcal{M} \subseteq$ Mono). Moreover, we will often work in categories in which each object is " $\lambda$-small" for some infinite regular cardinal $\lambda$. In the case of locally presentable categories, " $\lambda$-small" objects are just the $\lambda$-presentable ones. Recall that an object $A$ is $\lambda$-presentable if $\mathcal{A}[A,-]: \mathcal{A} \rightarrow$ Set preserves $\lambda$-filtered colimits. However Peter Freyd and Max Kelly used a different type of smallness, called bound:

2.1. Definition An object $A$ is said to have bound $\lambda$ (an infinite cardinal), if $\mathcal{A}[A,-]$ preserves $\lambda$-directed unions of $\mathcal{M}$-subobjects.

Explicitly: given a $\lambda$-directed collection of subobjects $m_{i}: M_{i} \rightarrow M \quad(i \in I)$ in $\mathcal{M}$ whose union is $M$, in the sense that the morphism $\left[m_{i}\right]: \coprod_{i \in I} M_{i} \rightarrow M$ lies in $\mathcal{E}$, then every morphism from $A$ to $M$ factors through $m_{i}$ for some $i \in I$.

2.2. Definition We call $\mathcal{A}$ locally $\mathcal{E}$-bounded (or locally bounded w.r.t. $(\mathcal{E}, \mathcal{M})$ ), provided that every object in $\mathcal{A}$ has a bound. Locally bounded means locally Epi-bounded.

2.3. Remark Locally $\mathcal{E}$-bounded categories with limits were considered by P. Freyd and M. Kelly in [9] for the study of the Orthogonal Subcategory Problem, although they did not give them a name. Later, M. Kelly [15] showed that the results of [9] remain valid without the assumption of the existence of limits.

2.4. Examples (a) Recall from [10] that $\mathcal{A}$ is called locally $\lambda$-presentable if it is cocomplete and has a set of $\lambda$-presentable objects whose closure under $\lambda$-filtered colimits is all of $\mathcal{A}$.

As noted in [9], all locally presentable categories are locally bounded w.r.t. the factorization system (StrongEpi,Mono). This is an easy consequence of the fact that in a locally $\lambda$-presentable category each $\lambda$-directed diagram with monomorphisms as connecting morphisms fulfils the following: (i) its colimit consists of monomorphisms, and (ii) for every compatible cocone of monomorphisms the induced factorizing morphism is monic; see 1.62 and 1.63 of [5]. We will prove below that although none of (i) and (ii) is true for strong monomorphisms in general, locally presentable categories are nevertheless locally bounded (i.e., w.r.t. the factorization system (Epi, StrongMono)). 
(b) The category Top of topological spaces and continuous functions is locally bounded (cf. [9]).

(c) Let $\mathcal{A}$ be locally bounded w.r.t. $(\mathcal{E}, \mathcal{M})$. Then every full $\mathcal{E}$-reflective subcategory $\mathcal{L}$ (i.e., one whose reflection maps lie in $\mathcal{E}$ ) is locally bounded with respect to the factorization system $\left(\mathcal{E}^{\prime}, \mathcal{M}^{\prime}\right)$, where $\mathcal{E}^{\prime}=\mathcal{E} \cap \operatorname{Mor} \mathcal{L}$ and $\mathcal{M}^{\prime}=\mathcal{M} \cap \operatorname{Mor} \mathcal{L}$. Since $\mathcal{L}$ is closed under $\mathcal{M}$-subobjects in $\mathcal{A}$, we obtain a factorization system $\left(\mathcal{E}^{\prime}, \mathcal{M}^{\prime}\right)$ in $\mathcal{L}$. Every object of $\mathcal{L}$ of bound $\lambda$ in $\mathcal{A}$ has bound $\lambda$ in $\mathcal{L}$ w.r.t. $\left(\mathcal{E}^{\prime}, \mathcal{M}^{\prime}\right)$. In fact, let $m_{i}: M_{i} \rightarrow M(i \in I)$ be morphisms in $\mathcal{M}^{\prime}$ having union $M$ in $\mathcal{L}$, then it is easy to see that they have union $M$ in $\mathcal{A}$ too. Thus, every morphism $L \rightarrow M$ in $\mathcal{L}$ factorizes through some $m_{i}$.

(d) The category Haus of Hausdorff topological spaces is locally bounded w.r.t. the factorization system (Surjections, Embeddings), see (c). However it is not locally bounded w.r.t. (StrongEpi, Mono) nor (Epi,StrongMono) ([9], 3.3.5). For the results presented below the natural factorization system is (Epi,StrongMono). That is the reason why we use simply the term "locally bounded" in this case. However, the category Haus demonstrates that we should not restrict ourselves to that factorization system.

2.5. Lemma For every locally presentable category $\mathcal{A}$, there exist (arbitrary large) cardinals $\lambda$ such that the category $\mathcal{A}^{\rightarrow}$ of all epimorphisms (as a full subcategory of the morphism category $\mathcal{A}^{\rightarrow}$ ) is locally $\lambda$-presentable, and the inclusion functor $\mathcal{A}^{\rightarrow} \hookrightarrow \mathcal{A}^{\rightarrow}$ preserves $\lambda$-filtered colimits and $\lambda$-presentable objects.

Proof (1) We can consider $\mathcal{A}^{\rightarrow}$ as the category of all models of the sketch consisting of a single pushout $\left.f\right|_{b \underset{i d_{b}}{\longrightarrow} b} ^{a \stackrel{f}{\longrightarrow} b} i d_{b}$ in the category $\mathcal{A}$. Consequently, $\mathcal{A}^{\rightarrow}$ is accessible (see Theorem 2.60 in [5]), and being closed under colimits in $\mathcal{A}^{\rightarrow}$, it is cocomplete. The local presentability of $\mathcal{A}^{\rightarrow}$ follows from 2.47 in $[5]$.

(2) Since $\mathcal{A}^{\rightarrow}$ is closed under colimits in $\mathcal{A}^{\rightarrow}$, by Theorem 2.19 of [5], there exists a (arbitrary large) regular cardinal $\lambda$ such that $\mathcal{A}^{\rightarrow}$ is locally $\lambda$-presentable and its embedding into $\mathcal{A}^{\rightarrow}$ preserves $\lambda$-filtered colimits and $\lambda$-presentable objects.

2.6. Theorem Every locally presentable category is locally bounded. 
Proof (1) Let $\lambda$ be a cardinal such that the statement of 2.5 holds. We prove that for $k \geq \lambda$, every $k$-directed union of strong monomorphisms is a colimit.

Let

$$
f_{i}: A_{i} \rightarrow B(i \in I)
$$

be a $k$-directed diagram of strong monomorphisms in $\mathcal{A}$. Denote by

$$
x_{i}: A_{i} \rightarrow A(i \in I)
$$

the colimit cocone of the diagram of all connecting strong monomorphisms, then the factorizing morphism

$$
f: A \rightarrow B \text { with } f_{i}=f \cdot x_{i}(i \in I)
$$

is an epimorphism. To conclude the proof we verify that $f$ is a split monomorphism. Then $B=\operatorname{colim} A_{i}$.

Express $f$ as a $k$-filtered colimit of $k$-presentable objects $g_{u}$ of $\mathcal{A}^{\rightarrow}$ with the colimit cocone

$$
\left(t_{u}, s_{u}\right):\left(T_{u} \stackrel{g_{u}}{\longrightarrow} S_{u}\right) \longrightarrow(A \stackrel{f}{\longrightarrow} B)(u \in U) .
$$

Since $\left(x_{i}, \operatorname{id}_{B}\right):\left(A_{i} \stackrel{f_{i}}{\longrightarrow} B\right) \longrightarrow(A \stackrel{f}{\longrightarrow} B)$ is a $k$-filtered colimit in $\mathcal{A}^{\rightarrow}$, for every $u \in U$ there exists $i(u) \in I$ such that $\left(t_{u}, s_{u}\right)$ factors through $\left(x_{i(u), \text { id }_{B}}\right)$ in $\mathcal{A}^{\rightarrow}$ :

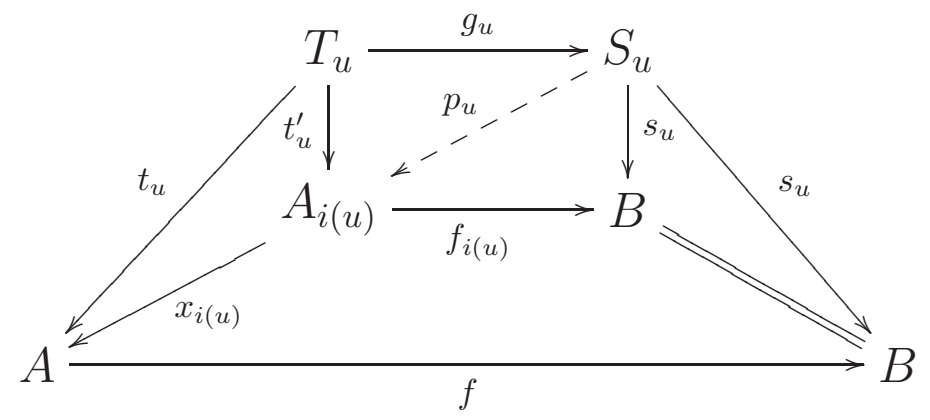

The diagonal fill-in property gives a morphism $p_{u}: S_{u} \rightarrow A_{i(u)}$ such that the above diagram commutes. It is easy to verify that the morphisms $x_{i(u)} \cdot p_{u}$ form a compatible cocone of the diagram of $S_{u}(u \in U)$, whose colimit is $B$ (use the fact that the morphisms $g_{u}$ are epimorphic); thus, we have a unique

$$
p: B \rightarrow A \text { with } p \cdot s_{u}=x_{i(u)} \cdot p_{u}(u \in U) .
$$


We conclude that

$$
p \cdot f \cdot t_{u}=t_{u} \text { for all } u \in U
$$

and since $\left(t_{u}\right)$ is a colimit cocone, we deduce $p \cdot f=\mathrm{id}$, as required.

(2) Every object $A$ is $k$-presentable for some regular cardinal $k$. Assuming (without loss of generality) that $k \geq \lambda$, it follows from (1) that $A$ has bound $k$ w.r.t. (Epi, StrongMono).

2.7. Definition ([13]) A morphism $f: A \rightarrow B$ of a category $\mathcal{A}$ is said to be $\lambda$-presentable, for an infinite regular cardinal $\lambda$, if it is a $\lambda$-presentable object in the slice category $A \downarrow \mathcal{A}$.

2.8. Examples (i) A morphism $f: A \rightarrow B$ in Set is finitely presentable iff it is

a. "almost epic", i.e., the set $B-f[A]$ is finite and

b. "almost monic", i.e., the set $\operatorname{ker} f-\Delta_{A}$ is finite.

(ii) Analogously, in the category of $R$-modules, an embedding $A \hookrightarrow B$ is finitely presentable iff the quotient $B / A$ is a finitely presentable module ([13]).

2.9. Facts The following facts will be used throughout. Where no reference is given, they can be easily verified.

(i) Every composition of two $\lambda$-presentable morphisms is $\lambda$-presentable. (Observe that if $f: A \rightarrow B$ and $g: B \rightarrow C$ are two $\lambda$-presentable morphisms, $x_{i}^{\prime}: x_{i} \rightarrow(A \stackrel{x}{\longrightarrow} X)$ are the colimit morphisms of a $\lambda$-filtered diagram $x_{i j}:\left(A \stackrel{x_{i}}{\longrightarrow} X_{i}\right) \longrightarrow\left(A \stackrel{x_{j}}{\longrightarrow} X_{j}\right)$, and $k: g \cdot f \rightarrow x$ is a morphism in $A \downarrow \mathcal{A}$, then there exists $i$ and a morphism $t$ such that $k \cdot g=x_{i}^{\prime} \cdot t$, and $k \cdot g$ is the colimit of a $\lambda$-filtered diagram $x_{j j^{\prime}}: x_{i j} \cdot t \rightarrow x_{i j^{\prime}} \cdot t$ in $B \downarrow \mathcal{A}$.)

(ii) Every morphism $f: A \rightarrow B$ with $A$ and $B \lambda$-presentable (in $\mathcal{A}$ ) is a $\lambda$-presentable morphism. (These are precisely the $\lambda$-presentable objects of $\mathcal{A}^{\rightarrow}$.) Conversely, if $f: A \rightarrow B$ and $A$ are $\lambda$-presentable, then $B$ is also $\lambda$ presentable. This implies that all $\lambda$-presentable morphisms with a common domain form essentially a set (since each object $A$ is $\gamma$-presentable for some $\gamma$ ). This is also a consequence of the fact that $A \downarrow \mathcal{A}$ is locally $\lambda$-presentable for every $A$, see [5].

(iii) In every pushout 


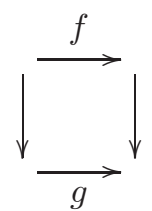

if $f$ is $\lambda$-presentable, then so is $g$. Theorem 2.3 of [13] shows that in locally $\lambda$ presentable categories, $\lambda$-presentable morphisms are actually characterized as the pushouts of the morphisms with $\lambda$-presentable domains and codomains.

2.10. Theorem Let $\mathcal{A}$ be a locally $\lambda$-presentable category. Then

(i) every strong epimorphism $f: A \rightarrow B$ is a $\lambda$-filtered colimit (in $A \downarrow \mathcal{A}$ ) of $\lambda$-presentable strong epimorphisms; and

(ii) there exists a regular cardinal $\bar{\lambda}$ such that every epimorphism $f: A \rightarrow B$ is a $\bar{\lambda}$-filtered colimit (in $A \downarrow \mathcal{A}$ ) of $\bar{\lambda}$-presentable epimorphisms.

Observe the difference between this theorem and Lemma 2.5: here we are working in the slice categories.

Proof (i) Since $A \downarrow \mathcal{A}$ is locally $\lambda$-presentable (see [5]), $f$ is the colimit of the canonical diagram made of all the $\lambda$-presentable morphisms with domain $A$ through which $f$ factors. Let $\left(l_{j}:\left(e_{j}, A_{j}\right) \rightarrow(e, C)\right)$ be the colimit diagram of the full subdiagram (also $\lambda$-filtered) of all $\lambda$-presentable strong epimorphisms. We show that the induced $g: C \rightarrow B$ is an isomorphism. Since it is certainly a strong epimorphism, we need only to show it is monomorphic. For that it suffices to check that it has the diagonal fill-in property with respect to strong epimorphisms between $\lambda$-presentable objects, see [12] or 5.7 of [7]. Let $q: X \rightarrow Y$ be a strong epimorphism with $X$ and $Y \lambda$-presentable, and let $u$ and $v$ be morphisms such that $v \cdot q=g \cdot u$. The $\lambda$-presentability of $X$ assures the existence of some morphism $\bar{u}: X \rightarrow A_{j}$ with $j \in J$ such that $u=l_{j} \cdot \bar{u}$. Let $(\hat{q}, \hat{u})$ be the pushout of $(q, \bar{u})$.

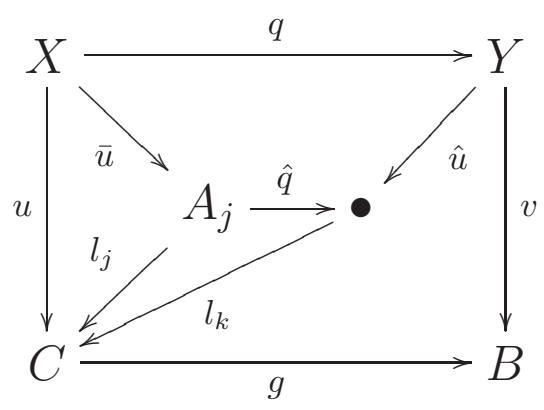

Then $\hat{q}$ is a $\lambda$-presentable strong epimorphism, and so is its composition with $e_{j}$. That is, $\hat{q} \cdot e_{j}=e_{k}$ for some $k \in J$. The equality $l_{k} \cdot \hat{q}=l_{j}$ follows because 
$e_{j}$ is an epimorphism. Thus, we easily conclude that $l_{k} \cdot \hat{u}$ is the desired diagonal morphism.

(ii) Choose a cardinal $\bar{\lambda} \geq \lambda$ such that Lemma 2.5 holds for it. We use the same argument of the proof of Proposition 1.2 of [12]. Given an epimorphism $f: A \rightarrow B$, let $\left(a_{i}, b_{i}\right): f_{i} \rightarrow f$ be the colimit morphisms of a $\bar{\lambda}$-filtered diagram $\left(f_{i} \rightarrow f_{j}\right)$, with all $f_{i} \bar{\lambda}$-presentable objects of $\mathcal{A}^{\rightarrow}$. If $f_{i}^{*}$ is the pushout of $f_{i}$ by $a_{i}$, then $f$ is a $\bar{\lambda}$-filtered colimit in $A \downarrow \mathcal{A}$ of the obvious diagram with objects the $f_{i}^{*}$. Since, by 2.5 , each $f_{i}$ is a $\bar{\lambda}$-presentable object in $\mathcal{A}^{\rightarrow}$, then, by 2.9 , the morphisms $f_{i}^{*}$ are $\bar{\lambda}$-presentable epimorphisms.

2.11. Remark In the statement (ii) of the preceding theorem, it is not always possible to choose $\bar{\lambda}=\lambda$. This is demonstrated by Example 1.5(b) of [12].

\section{The Small Object Argument}

We explained in the introduction that our aim is to extend the generalized Small Object Argument as formulated in [4], from sets to (certain) classes of morphisms. The statement of the Small Object Argument is that in every "reasonable" category $\mathcal{A}$, and given a set (or a "reasonable" class) $\mathcal{A}$ of morphisms, then

(1) $\operatorname{Inj} \mathcal{H}$ is a weakly reflective subcategory, and

(2) the weak reflection of every object can be chosen to be cellular (see below).

We are going to prove that if $\mathcal{A}$ is a locally bounded category, then (1) and (2) hold for classes $\mathcal{H}$ of morphisms such that all members but a subset are epimorphisms. This nicely corresponds to the result of Max Kelly [15] that under precisely the same assumptions,

\section{$\mathcal{H}^{\perp}$ is a reflective subcategory of $\mathcal{A}$.}

Indeed, we are going to simply modify the technique of using pointed endofunctors which Kelly employed brilliantly in his paper. He used well-pointed functors, that is, the given natural transformation $\sigma: I d \rightarrow S$ satisfied $S \sigma=\sigma S$. Our pointed functors are unfortunately not well-pointed, therefore, some modifications are necessary.

We also give a surprising negative result: although the "locally ranked" categories used in [4] are but a small variation of locally bounded ones, they cannot be used for the generalization above: in the last section, a counterexample is presented. 
3.1. Definition Given a class $\mathcal{H}$ of morphisms in $\mathcal{A}$, the Small Object Argument is valid, provided that every object of $\mathcal{A}$ has a weak reflection in $\operatorname{Inj} \mathcal{H}$ which lies in the class

\section{cell $\mathcal{H}$}

of $(\mathcal{H}-)$ cellular morphisms, that is, the closure of $\mathcal{H}$ under composition, pushout and wide pushout. Here, closing $\mathcal{H}$ under pushouts means adding every pushout of a member of $\mathcal{H}$ (by any morphism), and closing under wide pushouts means adding any wide pushout of a (small) family of members of $\mathcal{H}$ (that is, for any set of morphisms $h_{i}: A \rightarrow A_{i}, i \in I$, in $\mathcal{H}$, the coproduct in $A \downarrow \mathcal{A}$ of the objects $h_{i}$ is a morphism of $\left.\mathcal{H}\right)$.

The concept of cellular morphism plays an important role in Homotopy Theory. There, a different but equivalent formulation is used:

3.2. Definition Let $\alpha$ be an ordinal.

(1) A chain $C: \alpha \rightarrow \mathcal{A}$ is called smooth if it preserves directed colimits (that is, for every limit ordinal $i \in \alpha$, we have $C_{i}=\operatorname{colim}_{j<i} C_{j}$ ).

(2) A morphism $h$ is an $\alpha$-composite of morphisms $h_{i}(i<\alpha)$ provided that there exists a smooth chain $C: \alpha+1 \rightarrow \mathcal{A}$ such that $h_{i}=C_{i, i+1}$ for every $i<\alpha$ and $h=C_{0, \alpha}$. The case $\alpha=0$ expresses precisely that $h$ is an identity morphism, while the case $\alpha=2$ is the usual concept of composition.

(3) A transfinite composite is an $\alpha$-composite for some ordinal $\alpha$.

Given a class $\mathcal{H}$ of morphisms, it is easy to see that cell $\mathcal{H}$ is the closure of $\overline{\mathcal{H}}$ under transfinite composition, where $\overline{\mathcal{H}}$ is the closure of $\mathcal{H}$ under pushouts.

3.3. Terminology Let $\mathcal{A}$ be a category with a proper factorization system $(\mathcal{E}, \mathcal{M})$. A class of morphisms in $\mathcal{A}$ is called quasi-small if all members but a set lie in $\mathcal{E}$.

3.4. Construction Given a quasi-small class $\mathcal{H}$ in a (cocomplete and $\mathcal{E}$ cowellpowered) category $\mathcal{A}$, we define a pointed endofunctor

$$
\sigma: I d_{\mathcal{A}} \rightarrow S
$$


as follows: given an object $A$, consider all spans $(f, h)$, where $h \in \mathcal{H}$ and $f$ has codomain $A$. For each such $(f, h)$, form their pushout $\left(h_{f}, f^{\prime}\right)$

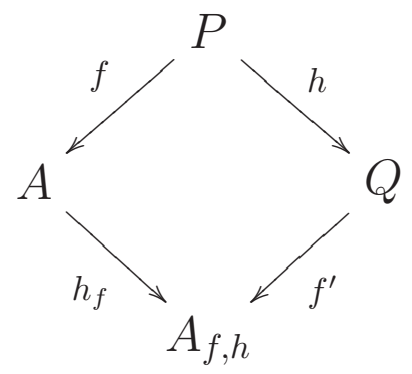

To define $S$ and $\sigma$ on the object $A$, form the wide pushout of all the $h_{f}$ : $A \rightarrow A_{f, h}$ as above:

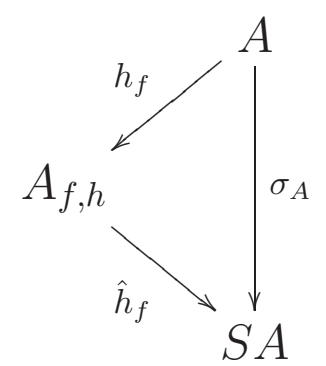

This pushout exists because $\mathcal{A}$ is $\mathcal{E}$-cowellpowered and all but a set of morphisms $h_{f}$ lie in $\mathcal{E}$. The definition of $S$ on morphisms $u: A \rightarrow A^{\prime}$ is obvious: $S u$ is the unique morphism such that $S u \cdot \hat{h}_{f}=\hat{h}_{u \cdot f} \cdot \bar{u}$, with $\bar{u}$ the pushout of $u$ along $h_{f}$.

3.5. Remark (i) Every object injective w.r.t. $\mathcal{H}$ is injective w.r.t. $\sigma_{A}$. This follows immediately from the definition of $\sigma_{A}$.

(ii) $A$ is injective w.r.t. $\mathcal{H}$ iff $\sigma_{A}$ is a split monomorphism.

3.6. Theorem In every locally $\mathcal{E}$-bounded category, the Small Object Argument is valid for all quasi-small classes.

Proof Let $\mathcal{H}=\mathcal{H}_{o} \cup \mathcal{H}_{e}$ be a class of morphisms with $\mathcal{H}_{o}$ small and $\mathcal{H}_{e} \subseteq \mathcal{E}$. For every object $A$ we present a weak reflection in $\operatorname{Inj} \mathcal{H}$.

(1) Let

$$
X: \text { Ord } \rightarrow \mathcal{A}
$$

be the chain of objects $X_{i}$ and morphisms $x_{i j}(i \leq j)$ defined by transfinite induction as follows:

$X_{0}=A$

$X_{i+1}=S X_{i}$ and $x_{i, i+1}=\sigma_{X_{i}} \quad$ for all ordinals $i$

$X_{i}=\operatorname{colim}_{j<i} X_{j}$ with the colimit cocone $x_{j i}$ for all limit ordinals $i$. 
Since we work in a cocomplete $\mathcal{E}$-cowellpowered $(\mathcal{E}, \mathcal{M})$-category, it follows from [17] 8.5 that there exist an embedding $\varphi$ : Ord $\rightarrow$ Ord preserving joins, a chain $Y:$ Ord $\rightarrow \mathcal{A}$, and a natural transformation

$$
\beta_{i}: Y_{i} \rightarrow X_{\varphi(i+1)} \quad(i \in \text { Ord })
$$

with the following properties (where we will use the notation $\hat{i}=\varphi(i+1)$ ):

(i) For every ordinal $i$, the composites

$$
Y_{i} \stackrel{\beta_{i}}{\longrightarrow} X_{\hat{i}} \stackrel{x_{\hat{i} j}}{\longrightarrow} X_{j}
$$

lie in $\mathcal{M}$ for all $j \geq \hat{i}$.

(ii) For every limit ordinal $i$, the cocone

$$
Y_{j} \stackrel{\beta_{j}}{\longrightarrow} X_{\hat{j}} \stackrel{x_{\hat{j} \varphi(i)}}{\longrightarrow} X_{\varphi(i)} \quad(j<i)
$$

is a colimit of the chain of all $Y_{j}$ for $j<i$.

(2) Since $\mathcal{H}_{o}$ is small, there exists a regular cardinal $k$ such that all domains and codomains of morphisms of $\mathcal{H}_{o}$ are bounded by $k$. We prove that if

$$
e: X_{\varphi(k)} \rightarrow Z \quad \text { is a morphism of } \mathcal{E}
$$

such that

$$
n_{i} \equiv\left(Y_{i} \stackrel{x_{\hat{\imath} \varphi(k)} \cdot \beta_{i}}{\longrightarrow} X_{\varphi(k)} \stackrel{e}{\longrightarrow} Z\right) \text { lies in } \mathcal{M}(i<k),
$$

then $Z$ is injective w.r.t. $\mathcal{H}_{o}$.

From (ii) of (1), one concludes that the morphisms

$$
Y_{i} \stackrel{\beta_{i}}{\longrightarrow} X_{\hat{i}} \stackrel{x_{\hat{i} \varphi(k)}}{\longrightarrow} X_{\varphi(k)}, i<k
$$

are the colimit morphisms of a $k$-directed colimit whose connecting morphisms lie in $\mathcal{M}$. Since $e$ belongs to $\mathcal{E}$, it follows that $Z$ is the union of the $\mathcal{M}$-subobjects $n_{i}: Y_{i} \rightarrow Z(i<k)$. Now, given $h: A \rightarrow B$ in $\mathcal{H}_{o}$ and a morphism $f: A \rightarrow Z$, since $A$ is bounded by $k$ there is some ordinal $i$ and some morphism $\bar{f}: A \rightarrow Y_{i}$ such that

$$
n_{i} \cdot \bar{f}=f .
$$


From the definition of $(S, \sigma)$ we see that there exists a commutative diagram of the form

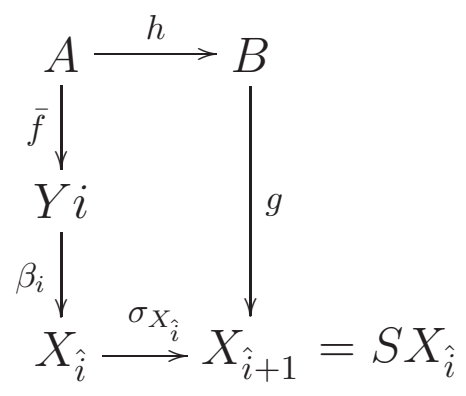

Consequently,

$$
\left(e \cdot x_{\hat{i}+1, \varphi(k)} \cdot g\right) \cdot h=e \cdot x_{\hat{i}+1, \varphi(k)} \cdot \sigma_{X_{\hat{i}}} \cdot \beta_{i} \cdot \bar{f}=e \cdot x_{\hat{i}, \varphi(k)} \cdot \beta_{i} \cdot \bar{f}=n_{i} \cdot \bar{f}=f .
$$

(3) By using $\mathcal{H}_{e}$ in place of $\mathcal{H}$, we obtain a new pointed endofunctor $(\bar{S}, \bar{\sigma})$ and the components of $\bar{\sigma}$ lie in $\mathcal{E}$. Let us define a chain $Z: \operatorname{Ord} \rightarrow \mathcal{A}$ analogous to the chain $X$ in (1) by putting $Z_{0}=X_{\varphi(k)}, Z_{i+1}=\bar{S} Z_{i}$ and $Z_{i}=\operatorname{colim}_{j<i} Z_{j}$ for limit ordinals $i$. Every morphism $z_{i j}$ (for $i \leq j$ ) of this chain belongs to $\mathcal{E}$. Since $\mathcal{E}$ is cowellpowered, the chain converges, i.e., there is some $l \in$ Ord such that $z_{l j}$ is an isomorphism for every $j \geq l$. We are going to show that $Z_{l}$ is $\mathcal{H}$-injective, which will finish the proof.

The object $Z_{l}$ is clearly $\mathcal{H}_{e}$-injective because $\bar{\sigma}_{Z_{l}}=z_{l, l+1}$ is an isomorphism. In order to show that $Z_{l}$ is $\mathcal{H}_{o}$-injective, we apply (2): it suffices to show that, for each $i<\varphi(k)$, the morphism

$$
Y_{i} \stackrel{x_{\hat{\imath}, \varphi(k)} \cdot \beta_{i}}{\longrightarrow} X_{\varphi(k)} \stackrel{z_{0 l}}{\longrightarrow} Z_{l}
$$

belongs to $\mathcal{M}$.

Observe that we have an obvious natural transformation $\alpha$ :

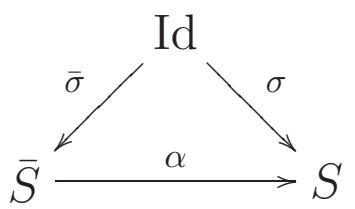

whose components are given by the unique factorization of a smaller wide pushout through a bigger one. This yields a natural transformation

$$
\gamma_{i}: Z_{i} \rightarrow X_{\varphi(k)+i}(i \in \text { Ord })
$$


given by $\gamma_{0}=\mathrm{id}_{Z_{0}}, \gamma_{i}=\operatorname{colim}_{j<i} \gamma_{j}$ for limit ordinals $i$, and

$$
\gamma_{i+1} \equiv\left(\bar{S} Z_{i} \stackrel{\alpha_{Z_{i}}}{\longrightarrow} S Z_{i} \stackrel{S \gamma_{i}}{\longrightarrow} S X_{\varphi(k)+i}=X_{\varphi(k)+i+1}\right) .
$$

This transformation satisfies $\gamma_{i} \cdot z_{0 i}=x_{\varphi(k), \varphi(k)+i}$ for all $i$ (as it is easily seen by induction). We obtain a commutative diagram

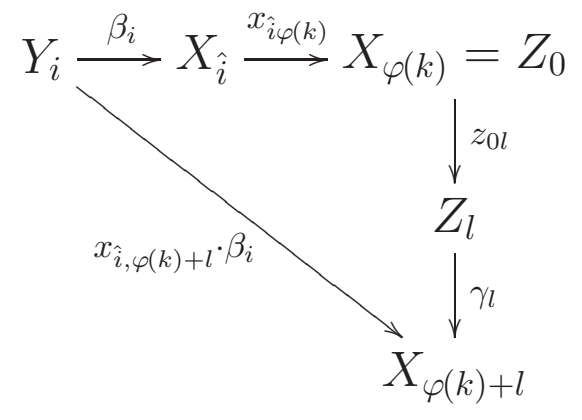

whose lower path lies in $\mathcal{M}$, thus, the morphism $z_{0 l} \cdot x_{\hat{i} \varphi(k)} \cdot \beta_{i}$ lies in $\mathcal{M}$. Consequently, (2) implies

$$
Z_{l} \in \operatorname{Inj} \mathcal{H} \text {. }
$$

(4) An easy transfinite induction shows that every object injective w.r.t. $\mathcal{H}$ is injective w.r.t. the $\mathcal{H}$-cellular morphisms $x_{0 \varphi(k)}: A \rightarrow X_{\varphi(k)}$ and $z_{0 l}$ : $X_{\varphi(k)} \rightarrow Z_{l}$, then injective w.r.t. the $\mathcal{H}$-cellular morphism

$$
r_{A} \equiv\left(A \stackrel{x_{0 \varphi(k)}}{\longrightarrow} X_{\varphi(k)} \stackrel{z_{0 l}}{\longrightarrow} Z_{l}\right) .
$$

Consequently, since $Z_{l}$ belongs to $\operatorname{Inj} \mathcal{H}, r_{A}$ is the desired weak reflection.

3.7. Remark Theorem 3.6 is completely analogous to the result of Peter Freyd and Max Kelly on the Orthogonal Subcategory Problem. There are, however, differences between that problem and the Small Object Argument. For example, if the set-theoretical Vopěnka's Principle is assumed, then for all (!) classes $\mathcal{H}$ of morphisms in locally presentable categories, the subcategory $\mathcal{H}^{\perp}$ is reflective, see [5]. In contrast, independently of set theory, there are situations where in an "everyday" locally finitely presentable category, the Small Object Argument fails:

3.8. Example of a class $\mathcal{H}$ of graph homomorphisms such that the Small Object Argument is not valid.

Here $\mathcal{A}$ is the (locally finitely presentable) category of graphs. Let $\mathcal{H}$ be the class of all

$$
0 \rightarrow C_{n} \quad(n \in \mathrm{Card})
$$


where 0 is the initial (empty) graph and $C_{n}$ is a clique on $n$ nodes (i.e., where two nodes form an edge iff they are distinct). It is rather obvious that

$\operatorname{Inj} \mathcal{H}=$ the class of all graphs with a loop.

Then, no nonempty graph without loops has a cellular weak reflection in Inj $\mathcal{H}$. In fact, this is proved by an analogous argument to that of Example 6.1 in $[2]$.

3.9. Definition A class $\mathcal{H}$ of morphisms is called

(i) presentable if there exists an infinite cardinal $\lambda$ such that all members of $\mathcal{H}$ are $\lambda$-presentable morphisms (see 2.7);

(ii) quasi-presentable if

$$
\mathcal{H}=\mathcal{H}_{0} \cup \mathcal{H}_{e} \text { with } \mathcal{H}_{0} \text { presentable and } \mathcal{H}_{e} \subseteq \text { Epi. }
$$

3.10. Remark A presentable class need not have, in any reasonable sense, a set of representatives: consider for example the classes $\mathcal{H}$ referred to in Example 2.8: the finitely presentable morphisms in Set, and the finitely presentable monomorphisms in $R$-modules. Note that, in the case of $R$ modules, $\operatorname{Inj}(\mathcal{H})$ turns out to be the class of all $F P$-injective (or absolutely pure) $R$-modules (see [14]).

3.11. Theorem In every locally presentable category the Small Object Argument is valid for all quasi-presentable classes of morphisms.

Proof Let $\mathcal{H}$ be a quasi-presentable class of morphisms in a locally presentable category $\mathcal{A}$. Then by Theorem 2.10, there exists a cardinal $\lambda$ such that

(i) $\mathcal{A}$ is locally $\lambda$-presentable,

(ii) every epimorphism with domain $X$ is a $\lambda$-filtered colimit of $\lambda$-presentable epimor-

phisms with domain $X$, and

(iii) all members of $\mathcal{H}$ are $\lambda$-presentable or epimorphisms.

We use a modification of the Construction 3.4:

For every object $A$, consider all $\lambda$-presentable morphisms $p: A \rightarrow P$ which have a "prolongation" $A \stackrel{p}{\longrightarrow} P \stackrel{q}{\longrightarrow} Q$ such that $q \cdot p$ is $\mathcal{H}$-cellular. Let

$$
\left\{p_{t}: A \rightarrow P_{t} ; t \in T_{A}\right\}
$$


be a set of representatives of all $\lambda$-presentable morphisms prolongable to $\mathcal{H}$-cellular morphisms. For each $t$, choose one

$$
q_{t}: P_{t} \rightarrow Q_{t} \text { with } q_{t} \cdot p_{t} \in \text { cell } \mathcal{H}
$$

We form the wide pushout

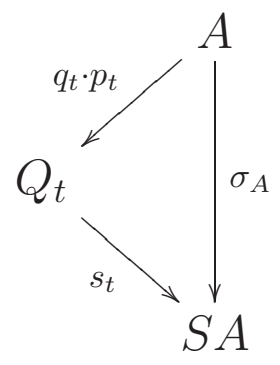

Since $\sigma_{A}$ is a wide pushout of cellular morphisms, it is cellular. We will iterate $\lambda$ times by defining a chain of objects $A_{i}(i<\lambda)$ and cellular morphisms

$$
r_{i j}: A_{i} \rightarrow A_{j} \quad \text { for } i \leq j \leq \lambda .
$$

We will then prove that

$$
r_{0, \lambda}: A \rightarrow A_{\lambda}
$$

is a weak reflection of $A$ in $\operatorname{Inj} \mathcal{H}$.

The chain is defined by transfinite induction:

First step: $A_{0}=A$.

Iterated step: $A_{i+1}=S A_{i}$ and $r_{i, i+1}=\sigma_{A_{i}}$.

Limit step: $A_{j}=\operatorname{colim}_{i<j} A_{i}$ for all limit ordinals $j \leq \lambda$ and $r_{i, j}(i<j)$ is the colimit cocone.

It is obvious that

$$
r_{i, j} \in \operatorname{cell} \mathcal{H} \text { for all } i, j,
$$

and it remains to show that $A_{\lambda} \in \operatorname{Inj} \mathcal{H}$ and $r_{0, \lambda}$ is a weak reflection of $A$. (a) Injectivity of $A_{\lambda}$ w.r.t. $\mathcal{H}$ :

Given a span $\left(f: X \rightarrow A_{\lambda}, h: X \rightarrow Y\right)$ with $h \in \mathcal{H}$, we prove that $f$ factors through $h$. Form a pushout $h^{\prime}$ of $h$ along $f$ :

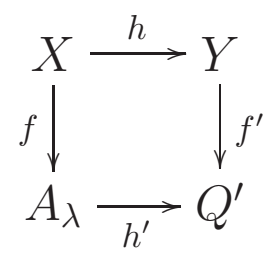


We know that $h$ is either $\lambda$-presentable or an epimorphism. In the latter case, express $h$ as a $\lambda$-filtered colimit of $\lambda$-presentable epimorphisms

$$
h_{d}: X \rightarrow Y_{d} \quad(d \in \mathrm{D})
$$

with the colimit cocone

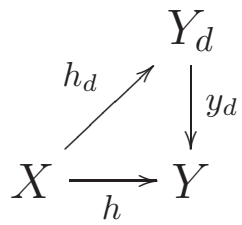

in $X \downarrow \mathcal{A}$. In the former case, just put $\mathrm{D}=\{d\}$ and $h=h_{d}$.

By 2.9(iii), the $\lambda$-presentable morphism $h_{d}$ is a pushout of some $h_{d}^{*}: X_{d}^{*} \rightarrow$ $Y_{d}^{*}$, where $X_{d}^{*}$ and $Y_{d}^{*}$ are $\lambda$-presentable objects:

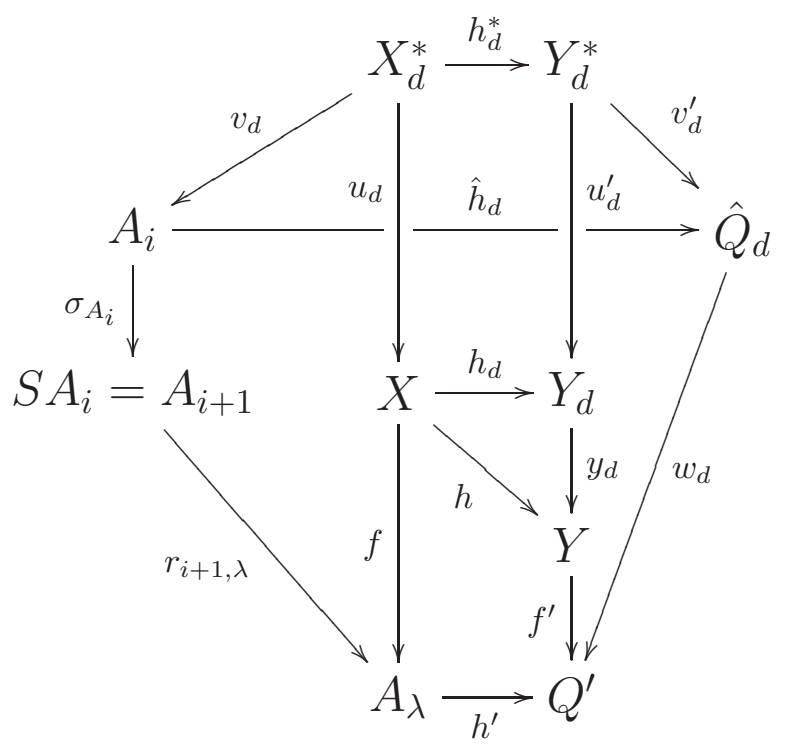

Since $X_{d}^{*}$ is $\lambda$-presentable, the morphism $f \cdot u_{d}: X_{d}^{*} \rightarrow A_{\lambda}=\operatorname{colim}_{i<\lambda} A_{i}$ factorizes through some of the colimit morphisms. That is, there exists an ordinal $i<\lambda$ (depending on $d$ ) and a morphism $v_{d}: X_{d}^{*} \rightarrow A_{i}$ such that

$$
f \cdot u_{d}=r_{i, \lambda} \cdot v_{d}=r_{i+1, \lambda} \cdot \sigma_{A_{i}} \cdot v_{d} .
$$

Next we form the pushout

$$
\hat{h}_{d}: A_{i} \rightarrow \hat{Q}_{d}
$$

of $h_{d}^{*}$ along $v_{d}$ and observe that, due to 2.9,

$$
\hat{h}_{d} \text { is a } \lambda \text {-presentable morphism. }
$$


The obvious factorization morphism $w_{d}: \hat{Q}_{d} \rightarrow Q^{\prime}$ is a prolongation of $\hat{h}_{d}$ to a cellular morphism:

$$
w_{d} \cdot \hat{h}_{d}=h^{\prime} \cdot r_{i, \lambda} \in \operatorname{cell} \mathcal{H} .
$$

Consequently, in the above definition of $S A_{i}$ we can assume that $\hat{h}_{d}$ is one of the morphisms $p_{t}$ :

$$
\hat{h}_{d}=p_{t} \text { for some } t \in T_{A_{i}} .
$$

This implies $\sigma_{A_{i}}=s_{t} \cdot q_{t} \cdot \hat{h}_{d}$, and by composing this equality with $r_{i+1, \lambda}$, we get

$$
r_{i, \lambda}=r_{i+1, \lambda} \cdot s_{t} \cdot q_{t} \cdot \hat{h}_{d}=f_{d} \cdot \hat{h}_{d}
$$

where $f_{d}=r_{i+1, \lambda} \cdot s_{t} \cdot q_{t}$. Thus,

$$
f \cdot u_{d}=r_{i, \lambda} \cdot v_{d}=f_{d} \cdot \hat{h}_{d} \cdot v_{d}=f_{d} \cdot v_{d}^{\prime} \cdot h_{d}^{*} .
$$

The universal property of the pushout $h_{d}$ ( of $h_{d}^{*}$ ) thus yields a unique factorization $z_{d}: Y_{d} \rightarrow A_{\lambda}$ :

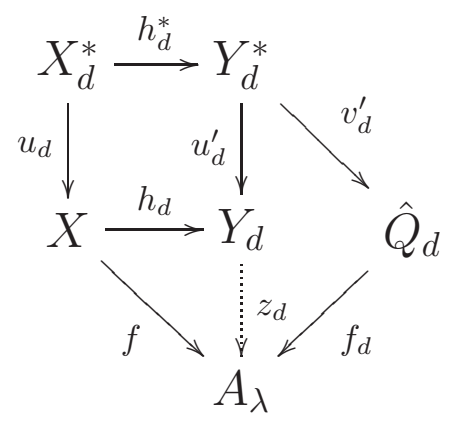

These morphisms are compatible, that is, for every connecting morphism $y_{d, d^{\prime}}: Y_{d} \rightarrow Y_{d^{\prime}}\left(d, d^{\prime} \in \mathrm{D}\right)$ of the diagram at the beginning, we have $z_{d}=$ $z_{d^{\prime}} \cdot y_{d, d^{\prime}}$. This is true by default if $\mathrm{D}=\{d\}$. If $h$ is an epimorphism, then $h_{d}$ is one, and we have

$$
z_{d} \cdot h_{d}=f=z_{d^{\prime}} \cdot h_{d^{\prime}}=\left(z_{d^{\prime}} \cdot y_{d, d^{\prime}}\right) \cdot h_{d}
$$

This yields a unique

$$
y: Y \rightarrow A_{\lambda} \text { with } y \cdot y_{d}=z_{d} \text { for } d \in \mathrm{D} .
$$

This is the desired factorization of $f$ through $h$ :

$$
f=z_{d} \cdot h_{d}=y \cdot y_{d} \cdot h_{d}=y \cdot h
$$


(b) Every morphism $b: A \rightarrow B$ with $B \in \operatorname{Inj} \mathcal{H}$ factorizes through $r_{0, \lambda}$. In fact, we find a compatible cocone $b_{i}: A_{i} \rightarrow B$ of the diagram defining $A_{\lambda}$ (with $b_{o}=b$ ), and then $b=b_{\lambda} \cdot r_{0, \lambda}$. The limit steps $b_{j}: \operatorname{colim}_{i<j} A_{i} \rightarrow B$ are clear from $A_{j}=\operatorname{colim}_{i<j} A_{i}$. For the isolated steps, just observe that since $B$ is injective w.r.t. $\mathcal{H}$, it is injective w.r.t. $\sigma_{A_{i}}$ for every $i$.

\section{Injectivity Logic}

The logic of injectivity concerns the relation

$$
\mathcal{H} \models k
$$

(injectivity consequence)

which states that every object injective w.r.t. $\mathcal{H}$ is also injective w.r.t. $k$. We will use the deduction system introduced in [8] as a slight modification of the deduction system of Grigore Roçu [21]. In our previous paper [1] we proved the completeness of the injectivity logic for all quasi-small classes of morphisms. However, the assumptions on the base category were far less pleasant than local boundedness. And also the proof was unnecessarily long. Here we are going to derive the completeness of the logic rather easily from the Small Object Argument.

We also present a new completeness result for locally presentable categories: the logic is complete for all quasi-presentable classes.

4.1. Definition The Injectivity Deduction System for morphisms $s, t, \ldots$ of a cocomplete category consists of the three deduction rules:

$$
\begin{array}{lll}
\text { PUShOUT } & \frac{s}{t} & \text { for every pushout } \\
\text { TRANSFINITE } & \frac{s_{i}(i<\alpha)}{t} & \begin{array}{l}
\text { if } t \text { is an } \alpha \text {-composite of the } \\
\text { COMPOSITION }
\end{array} \\
\text { CANCELLATION } & \frac{s \cdot t}{t}
\end{array}
$$

Given a class $\mathcal{H}$ of morphisms we say that a morphism $k$ is provable from it, notation

$$
\mathcal{H} \vdash k
$$


if there exists a formal proof of $k$ using as assumptions elements of $\mathcal{H}$ and using the Injectivity Deduction System.

4.2. Remark The Injectivity Logic is infinitary, thus, proofs have in general an ordinal length. A proof of $k$ from $\mathcal{H}$ of length $\beta$ is a sequence of morphisms

$$
k_{i}(i \leq \beta)
$$

such that $k=k_{\beta}$ and for each $i \leq \beta$, either

$k_{i} \in \mathcal{H}\left(k_{i}\right.$ is an assumption) or

$k_{i}$ is the conclusion of one of the three deduction rules whose assumption(s) lie(s) in $\left\{k_{j}\right\}_{j<i}$.

4.3. Corollary In locally $\mathcal{E}$-bounded categories, the Injectivity Logic is sound and complete for quasi-small classes $\mathcal{H}$ of morphisms. That is, given a morphism $k$ then

$$
\mathcal{H} \models k \quad \text { iff } \quad \mathcal{H} \vdash k .
$$

Proof Let $\mathcal{H} \models k: A \rightarrow B$, and let $r_{A}: A \rightarrow \bar{A}$ be an $\mathcal{H}$-cellular weak reflection in $\operatorname{Inj} \mathcal{H}$, which exists by 3.6. Then there exists $t: B \rightarrow \bar{A}$ such that $t \cdot k=r_{A}$. Being $\mathcal{H}$-cellular, $r_{A}$ is provable from $\mathcal{H}$, hence via CANCELlation, we derive $k$.

It is clear that, arguing as in 4.3, we obtain, as a consequence of Theorem 3.11, the completeness of the Injectivity Logic for quasi-presentable classes in locally presentable categories. In 4.4 we give a different proof of this fact, showing that, moreover, there is a functorial weak reflection for Inj $\mathcal{H}$.

4.4. Theorem For quasi-presentable classes $\mathcal{H}$ of morphisms in locally presentable categories:

(i) Inj $\mathcal{H}$ has a functorial weak reflection;

(ii) the Injectivity Logic is sound and complete.

Proof (i) Let $\mathcal{A}$ be a locally $\lambda$-presentable category and $\mathcal{H}$ a class of $\lambda$ presentable morphisms in $\mathcal{A}$. For each $A \in \mathcal{A}$, we construct a weak reflection $r_{A}$ in Inj $\mathcal{H}$ such that $\mathcal{H} \vdash r_{A}$ as follows. We define $r_{A}=r_{0, \lambda}$ for the chain of morphisms $r_{i, j}: A_{i} \rightarrow A_{j}$, all provable from $\mathcal{H}$, defined by the following transfinite induction: 
First step: $A_{0}=A$;

Isolated step: $r_{i, i+1}: A_{i} \rightarrow A_{i+1}$ is the wide pushout of all $\lambda$-presentable morphisms with domain $A_{i}$ provable from $\mathcal{H}$, and $r_{j, i+1}=r_{i, i+1} \cdot r_{j, i}$; it exists due to 2.7 .

Limit step: Given a limit ordinal $i$, then $r_{j, i}$ is the colimit of the chain $\left(r_{j, k}\right)_{k<i}$.

We put $r_{A}=r_{0, \lambda}$. It is clear that $\mathcal{H} \vdash r_{A}$, and it remains to show that $A_{\lambda}$ belongs to Inj $\mathcal{H}$.

Let $h: X \rightarrow Y$ belong to $\mathcal{H}$. Since $h$ is $\lambda$-presentable, there exist a morphism $h^{\star}: X^{\star} \rightarrow Y^{\star}$ with $\lambda$-presentable domain and codomain such that $h$ is the pushout of $h^{\star}$ along some morphism $u$ (see 2.9(iii)), as illustrated by the left-hand square below. Given $f: X \rightarrow A_{\lambda}$, it is our task to prove that $f$ factorizes through $h$. In fact let $h^{\prime}$ be the pushout of $h$ along $f$ as in the right-hand square:

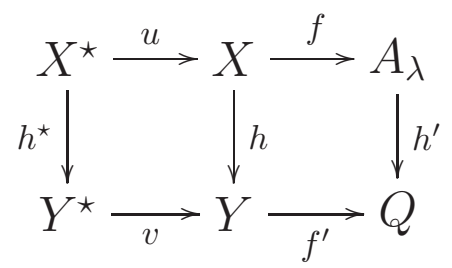

Since $X^{\star}$ is $\lambda$-presentable, there exists a morphism $g: X^{\star} \rightarrow A_{i}$, for some $i<\lambda$, such that $r_{i, \lambda} \cdot g=f \cdot u$. Let $p$ be the pushout of $h^{\star}$ along $g$, and consider the pushout $h^{\prime}$ of $p$ along $r_{i, \lambda}$; since $r_{i, \lambda} \cdot g=f \cdot u$, we conclude that $h^{\prime}$ is also the pushout of $h^{\star}$ along $f \cdot u$ :

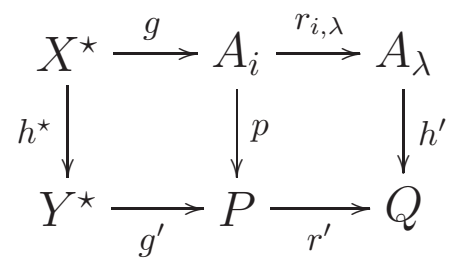

Taking into account that $r_{i, \lambda}$ and $h^{\prime}$ are both provable from $\mathcal{H}$, we have that $\mathcal{H} \vdash h^{\prime} \cdot r_{i, \lambda}=r^{\prime} \cdot p$. Being the pushout of $r_{i, \lambda}$, the morphism $r^{\prime}$ is also provable from $\mathcal{H}$, therefore, by applying CANCELLATION, we get $\mathcal{H} \vdash p$. Furthermore, the morphism $p$ is $\lambda$-presentable, because it is the puhout of $h^{\star}$. Hence $p$ is one of the branches forming the wide pushout $r_{i, i+1}: A_{i} \rightarrow A_{i+1}$. Consequently, there is a factorization $r_{i, i+1}=s \cdot p$. This yields

$$
1_{A_{\lambda}} \cdot(f \cdot u)=r_{i, \lambda} \cdot g=r_{i+1, \lambda} \cdot r_{i, i+1} \cdot g=r_{i+1, \lambda} \cdot s \cdot p \cdot g=\left(r_{i+1, \lambda} \cdot s \cdot g^{\prime}\right) \cdot h^{\star} .
$$


By the universality of the pushout $Q$, there exists a morphism $t: Q \rightarrow A_{\lambda}$ such that $t \cdot h^{\prime}=1_{A_{\lambda}}$. Thus, we get the desired factorization: $f=t \cdot h^{\prime} \cdot f=$ $\left(t \cdot f^{\prime}\right) \cdot h$.

Now, given $\mathcal{H} \models k$, we see that $r_{A}$ factorizes through $k$, then $\mathcal{H} \vdash r_{A}$ implies $\mathcal{H} \vdash k$ by CANCELLATION.

Finally, the functoriality of $r_{A}$ is obvious from its construction.

(ii) Let $\mathcal{H}$ be a quasi-presentable class, and choose a regular cardinal $\lambda$ such that (a) $\mathcal{A}$ is locally $\lambda$-presentable, (b) $\mathcal{H}=\mathcal{H}_{0} \cup \mathcal{H}_{e}$ with $\mathcal{H}_{0} \lambda$-presentable and $\mathcal{H}_{e} \subseteq E p i$ and (c) every epimorphism of $\mathcal{A}$ is expressible as a $\lambda$-filtered colimit of $\lambda$-presentable epimorphisms, see Theorem 2.6. For each $f \in \mathcal{H}_{e}$ select a family of $\lambda$-presentable epimorphisms of which $f$ is a colimit. Let $\hat{\mathcal{H}}_{e}$ be the union of all of these families. Then it is easy to see that Inj $\mathcal{H}_{e}=\operatorname{Inj} \hat{\mathcal{H}}_{e}$. Consequently the result follows from (i) applied to the $\lambda$-presentable class $\mathcal{H}_{o} \cup \hat{\mathcal{H}}_{e}$.

4.5. Example of a class of morphisms in a locally presentable category for which the Injectivity Logic is incomplete. Recall the example of cliques in graphs (3.8). Since every graph in $\operatorname{Inj} \mathcal{H}$ has a loop we have

$$
\mathcal{H} \models k \quad \text { for the unique } k: 0 \rightarrow 1 \text {. }
$$

We showed in [1] that, however, $k$ cannot be proved from $\mathcal{H}$.

\section{The Orthogonal Subcategory Problem and its Logic}

The Orthogonal Subcategory Problem asks whether, given a collection $\mathcal{H}$ of morphisms, the orthogonality class $\mathcal{H}^{\perp}$ is reflective, where $\mathcal{H}^{\perp}$ is the full subcategory of all objects orthogonal to every member of $\mathcal{H}$. From Max Kelly, we know that the answer is affirmative for all quasi-small classes $\mathcal{H}$ :

5.1. Theorem (see [15], 10.1 and 10.2) In every locally $\mathcal{E}$-bounded category the Orthogonal Subcategory Problem has an affirmative answer for all quasismall classes of morphisms.

5.2. Remark (i) In locally presentable categories, under the set-theoretic Weak Vopěnka's Principle, the Orthogonal Subcategory Problem has an affirmative answer for every class $\mathcal{H}$ (see Section 7 for more on that). We now prove that even without any large-cardinal set-theoretic assumption, the Orthogonal Subcategory Problem is affirmative if $\mathcal{H}$ is quasi-presentable.

(ii) In the locally bounded category Top there exist classes of morphisms for which the Orthogonal Subcategory Problem has a negative answer. In 
fact, by [6] there are full reflective subcategories $\mathcal{A}, \mathcal{B}$ of Top such that $\mathcal{A} \cap \mathcal{B}$ is not reflective. Let then $\mathcal{H}_{a}$ be the class of all reflection morphisms in $\mathcal{A}$, analogously for $\mathcal{H}_{b}$. Then $\left(\mathcal{H}_{a} \cup \mathcal{H}_{b}\right)^{\perp}$ is not reflective.

5.3. Theorem In a locally presentable category, $\mathcal{H}^{\perp}$ is reflective for every quasi-presentable class $\mathcal{H}$.

Proof (1) If $\mathcal{H}$ is presentable, then $\mathcal{H}^{\perp}$ is reflective by Proposition 3.16 of [2].

(2) Let $\mathcal{H}$ be a quasi-presentable class, and choose a regular cardinal $\lambda$ such that (i) $\mathcal{A}$ is locally $\lambda$-presentable, (ii) $\mathcal{H}=\mathcal{H}_{0} \cup \mathcal{H}_{e}$ with $\mathcal{H}_{0}$ formed by $\lambda$-presentable morphisms and $\mathcal{H}_{e} \subseteq E p i$, and (iii) every epimorphism of $\mathcal{A}$ is expressible as a $\lambda$-filtered colimit of $\lambda$-presentable epimorphisms, see Theorem 2.10. For each $f \in \mathcal{H}_{e}$ select a family of $\lambda$-presentable epimorphisms of which $f$ is a $\lambda$-filtered colimit. Let $\hat{\mathcal{H}}_{e}$ be the union of all of these families. Then it is easy to see that $\mathcal{H}_{e}^{\perp}=\hat{\mathcal{H}}_{e}^{\perp}$. Consequently the result follows from (1) applied to the presentable class $\mathcal{H}_{0} \cup \hat{\mathcal{H}}_{e}$.

5.4. Remark In [2] we formulated an Orthogonality Logic which studies orthogonality consequences of a class $\mathcal{H}$ of morphisms. This means those morphisms $k$ such that every object orthogonal to $\mathcal{H}$ is also orthogonal to $k$. We improve the results of [2] by proving that the Orthogonality Logic is complete for

(i) all quasi-small classes in locally bounded categories and

(ii) all quasi-presentable classes in locally presentable categories.

Throughout this section, $\models$ denotes the orthogonality consequence and $\vdash$ denotes provability in the following deduction system, presented in [2]:

5.5. Orthogonality Deduction System consists of four deduction rules: 
PUSHOUT $\quad \frac{s}{t}$

COEQUALIZER $\frac{s}{t}$ for every pushout $\underset{t}{\stackrel{s}{\longrightarrow} \downarrow}$

if $\stackrel{f}{\longrightarrow} \stackrel{t}{\longrightarrow}$ is a coequalizer

$$
f \cdot s=g \cdot s
$$

TRANSFINITE $\frac{s_{i}(i<\alpha)}{t}$ if $t$ is an $\alpha$-composite of the $s_{i}$ 's COMPOSITION

$$
\frac{u \cdot t \quad v \cdot u}{t}
$$
(see 3.2)

CANCELLATION

5.6. Remark The Orthogonality Logic is sound in every category with colimits (for all classes $\mathcal{H}$ ). That is,

$$
\mathcal{H} \vdash k \quad \text { implies } \quad \mathcal{H} \models k
$$

see $[2]$.

5.7. Theorem In a locally bounded category the Orthogonality Logic is complete for all quasi-small classes.

Proof (1) For every morphism $k: M \rightarrow N$, recall from [15] the pointed endofunctor $\sigma: I d \rightarrow S$ of $\mathcal{A}$ defined on objects $X$ by using the following pushout

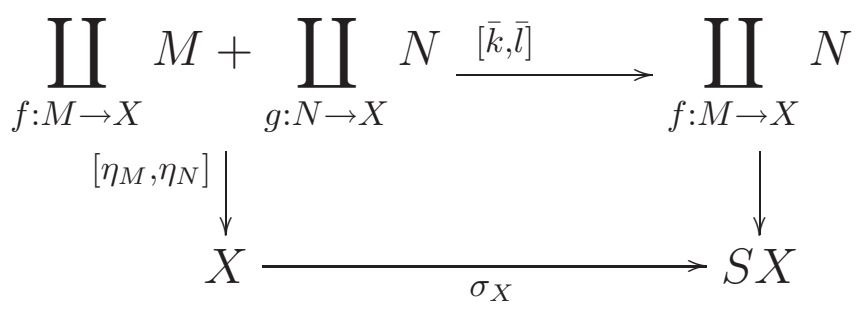

Here $\eta_{M}$ and $\bar{k}$ have their $f$-component equal to $f$ and to $k \cdot i_{f}$, respectively, where $i_{f}$ is the injection indexed by $f$, and $\eta_{N}$ and $\bar{l}$ have their $g$-component $g$ and the injection $i_{g \cdot k}$, respectively. We will prove that $\sigma_{X}$ is an orthogonality consequence of $k$ :

$$
k \vdash \sigma_{X}
$$


This is done by describing the above pushout as a certain wide pushout and observing that wide pushouts are transfinite composites of (ordinary) pushouts. For every morphism $f: M \rightarrow X$ form the pushout

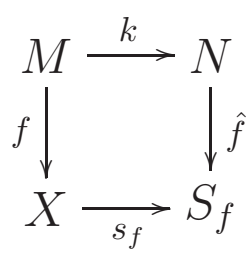

We have of course $k \vdash s_{f}$. Given a morphism $g: N \rightarrow X$ with $f=g \cdot k$, form a coequalizer

$$
N \underset{s_{f} \cdot g}{\stackrel{\hat{f}}{\longrightarrow}} S_{f} \stackrel{c_{f, g}}{\longrightarrow} C_{f, g}
$$

Since

$$
\hat{f} \cdot k=s_{f} \cdot g \cdot k
$$

we have, due to COEQUALIZER,

$$
k \vdash c_{f, g}
$$

Thus, if $c_{f}=a_{g} \cdot c_{f, g}: S_{f} \rightarrow C_{f}$ is the wide pushout of all $c_{f, g}$, where $g$ ranges over all the morphisms with $f=g \cdot k$, then

$$
k \vdash c_{f},
$$

and, consequently,

$$
k \vdash c_{f} \cdot s_{f} .
$$

Let us form a wide pushout of these morphisms

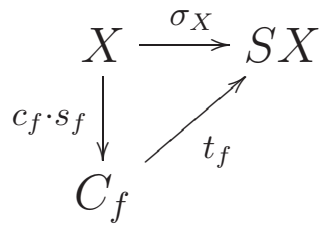

Then, once again,

$$
k \vdash \sigma_{X}
$$


We claim that the square

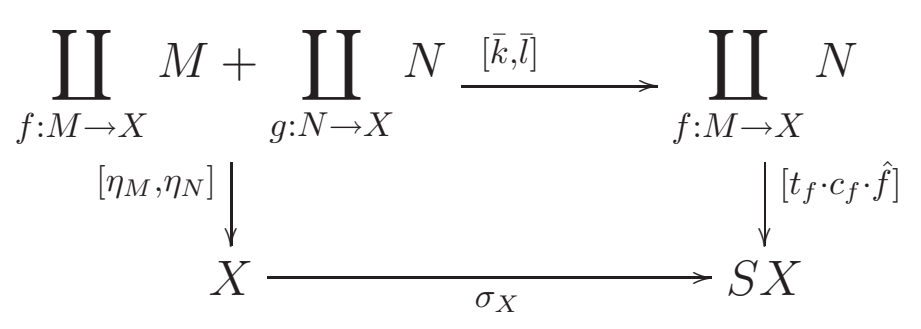

is a pushout:

(a) The square above commutes. In fact, the $f$-component commutes since

$$
\begin{aligned}
\sigma_{X} \cdot f & =t_{f} \cdot c_{f} \cdot s_{f} \cdot f \\
& =t_{f} \cdot c_{f} \cdot \hat{f} \cdot k .
\end{aligned}
$$

The $g$-component commutes because for $f=g \cdot k$ we have that

$$
\begin{aligned}
\sigma_{X} \cdot g & =t_{f} \cdot c_{f} \cdot s_{f} \\
& =t_{f} \cdot a_{g} \cdot c_{f, g} \cdot s_{f} \cdot g \\
& =t_{f} \cdot a_{g} \cdot c_{f, g} \cdot \hat{f} \\
& =t_{f} \cdot c_{f} \cdot \hat{f} .
\end{aligned}
$$

(b) Suppose that the square

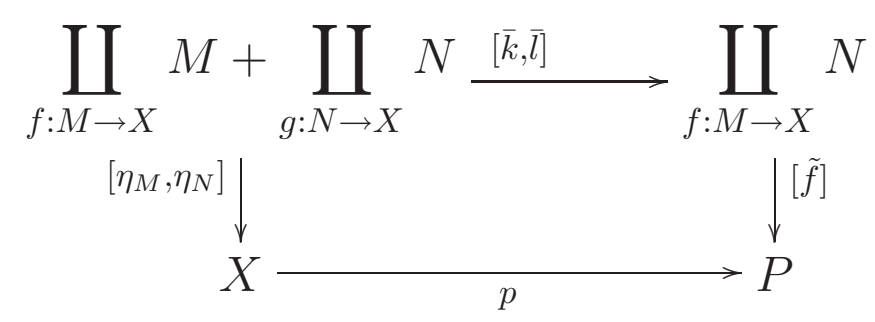

commutes. That is, to every $f: M \rightarrow X$ a morphism $\tilde{f}: N \rightarrow P$ is assigned with

$$
p \cdot f=\tilde{f} \cdot k
$$

and

$$
p \cdot g=\tilde{f}=\widetilde{g k} \quad \text { for } f=g \cdot k .
$$

For each $f$ we have a unique $u_{f}: S_{f} \rightarrow P$ with

$$
u_{f} \cdot s_{f}=p \quad \text { and } \quad u_{f} \cdot \hat{f}=\tilde{f} .
$$

Given $g$ with $f=g \cdot k$, then

$$
u_{f} \cdot \hat{f}=u_{f} \cdot s_{f} \cdot g
$$

because the left-hand side is $\tilde{f}$, and the right-hand one $p \cdot g=\widetilde{g \cdot k}=\tilde{f}$. 
Thus we get a unique $b_{g}: C_{f, g} \rightarrow P$ with $u_{f}=b_{g} \cdot c_{f, g}$ and this yields a unique

$$
v_{f}: C_{f} \rightarrow P \quad \text { with } \quad v_{f} \cdot a_{g}=b_{g}
$$

Since

$$
\begin{aligned}
v_{f} \cdot c_{f} \cdot s_{f} & =v_{f} \cdot a_{g} \cdot c_{f, g} \cdot s_{f} \\
& =b_{g} \cdot c_{f, g} \cdot s_{f} \\
& =u_{f} \cdot s_{f} \\
& =p
\end{aligned}
$$

we obtain a unique $v: S X \rightarrow P$ with

$$
v_{f}=v \cdot t_{f} \quad \text { and } \quad p=v \cdot \sigma_{X} .
$$

This morphism has then the desired properties:

$$
p=v \cdot \sigma_{X} \quad \text { and } \quad \tilde{f}=v \cdot\left(t_{f} \cdot c_{f} \cdot \hat{f}\right) \quad \text { for all } f: M \rightarrow X ;
$$

the latter equality follows because

$$
\tilde{f}=u_{f} \cdot \hat{f}=b_{g} \cdot c_{f, g} \cdot \hat{f}=v_{f} \cdot a_{g} \cdot c_{f, g} \cdot \hat{f}=v_{f} \cdot c_{f} \cdot \hat{f}=v \cdot t_{f} \cdot c_{f} \cdot \hat{f} .
$$

Conversely, these two properties determine $v$ uniquely.

(2) From the proof of Theorem 10.2 in [15] it follows that for every object $A$ of $\mathcal{A}$ the reflection $r_{A}: A \rightarrow \bar{A}$ exists "constructively", where the construction uses transfinite composition and wide pushouts of morphisms of the form $\sigma_{X}$ for various members $k \in \mathcal{H}$ and various objects $X$ of $\mathcal{A}$. Thus, (1) implies that $\mathcal{H} \vdash r_{A}$.

(3) We are ready to prove the completeness of the Orthogonality Logic for $\mathcal{H}$. Suppose that $k: A \rightarrow B$ is an orthogonality consequence of $\mathcal{H}$, and let $r_{A}: A \rightarrow \bar{A}$ be a reflection in $\mathcal{H}^{\perp}$. Then $\bar{A}$ is orthogonal to $r_{A}$ (since $\mathcal{H} \models r_{A}$ ) which yields $u: B \rightarrow A$ with $r_{A}=u \cdot k$. Due to (2), we have

$$
\mathcal{H} \vdash u \cdot k \text {. }
$$

Since $\mathcal{H} \models u \cdot k$ and $\mathcal{H} \models k$ we easily deduce $\mathcal{H} \models u$. Thus arguing as above with $u$ in the role of $k$, we conclude that there exists $v$ with

$$
\mathcal{H} \vdash v \cdot u \text {. }
$$

Then we obtain $\mathcal{H} \vdash k$ by WEAK CANCELLATION.

5.8. Example For general classes of morphisms in locally bounded categories the Orthogonality Logic is not complete in general. In fact, let $\mathcal{K}$ be the category of all triples $(A, \alpha, a)$ where $(A, \alpha)$ is a topological space and $a \subseteq$ $A \times A$ is a binary relation (a directed graph). Morphisms are all continuous 
graph homomorphisms. It is easy to see, arguing as in Top, that $\mathcal{K}$ is locally bounded. For the unique morphism

$$
v: 0 \rightarrow 1
$$

from the initial (empty) object 0 to the terminal (singleton loop) object 1 we present a class $\mathcal{H}$ such that

$$
\mathcal{H} \models v \quad \text { but } \quad \mathcal{H} \nvdash v .
$$

We use the result of Václav Koubek [16] that Top is almost universal. In particular there exists a proper class of topological spaces $\left(A_{i}, \alpha_{i}\right), i \in I$, such that there are no continuous maps between them except the constant maps and the identity self-maps. For each $i$ let $a_{i}$ be the clique on $A_{i}$ :

$$
a_{i}=A_{i} \times A_{i}-\Delta_{A_{i}} .
$$

This defines a morphism

$$
h_{i}: 0 \rightarrow\left(A_{i}, \alpha_{i}, a_{i}\right)
$$

in $\mathcal{K}$ and we put

$$
\mathcal{H}=\left\{h_{i}\right\}_{i \in I} .
$$

(1) We prove $\mathcal{H} \models v$, that is, whenever an object $(B, \beta, b)$ is orthogonal to $\mathcal{H}$, then the graph contains a unique loop. In fact, choose $i$ such that $A_{i}$ has cardinality bigger than $B$. Since $h_{i} \perp(B, \beta, b)$, we have a unique morphism $f:\left(A_{i}, \alpha_{i}, a_{i}\right) \rightarrow(B, \beta, b)$. There exists $x \neq x^{\prime}$ in $A_{i}$ with $f(x)=f\left(x^{\prime}\right)$. Since $x a_{i} x^{\prime}$, we conclude that $f(x)=f\left(x^{\prime}\right)$ is a loop. The loop is unique because every loop defines a constant morphism $\left(A_{i}, \alpha_{i}, a_{i}\right) \rightarrow(B, \beta, b)$.

(2) $\mathcal{H} \nvdash v$. Assuming the contrary we derive a contradiction: it is sufficient to find a category $\overline{\mathcal{K}}$ in which $\mathcal{K}$ is a full subcategory closed under colimits and such that

$$
\mathcal{H} \forall v \quad \text { in } \overline{\mathcal{K}}
$$

In fact, then a proof of $v$ from $\mathcal{H}$ in $\mathcal{K}$ is also a proof of $v$ from $\mathcal{H}$ in $\overline{\mathcal{K}}$, in contradiction to Remark 5.6.

We extend $\mathcal{K}$ by a single new object $\bar{K}$ : the intention is that $\bar{K}$ is the coproduct of all $\left(A_{i}, \alpha_{i}, a_{i}\right)$. Therefore, the morphisms of $\overline{\mathcal{K}}$ are

(a) all morphisms of $\mathcal{K}$;

(b) endomorphisms of $\bar{K}$ are precisely all families $\left(f_{i}\right)_{i \in I}$ where $f_{i}:\left(A_{i}, \alpha_{i}, a_{i}\right) \rightarrow$ $\left(A_{i^{\prime}}, \alpha_{i^{\prime}}, a_{i^{\prime}}\right)$ is a morphism of $\mathcal{K}$ for some $i^{\prime} \in I$ (recall that, then, $f_{i}$ is constant, or $i=i^{\prime}$ and $\left.f_{i}=\mathrm{id}\right)$; 
(c) analogously, morphisms from $\bar{K}$ to $(B, \beta, b) \in \mathcal{K}$ are precisely all families $\left(f_{i}\right)_{i \in I}$ of morphisms $f_{i}:\left(A_{i}, \alpha_{i}, a_{i}\right) \rightarrow(B, \beta, b)$ in $\mathcal{K}$ and

(d) morphisms from $(B, \beta, b)$ in $\mathcal{K}$ to $\bar{K}$ are precisely the morphisms

$$
f:(B, \beta, b) \rightarrow \coprod_{i \in J}\left(A_{i}, \alpha_{i}, a_{i}\right)
$$

where $J \subseteq I$ is a set; to make these well defined we assume that $J$ is the least such set, i.e., every $j \in J$ fulfils

$$
A_{j} \cap f[B] \neq \emptyset .
$$

The composition in $\overline{\mathcal{K}}$ is obvious: it is easy to see that $\mathcal{K}$ is a full subcategory of $\overline{\mathcal{K}}$ closed under colimits. We have

$$
\bar{K} \in \mathcal{H}^{\perp} \text {, but } \bar{K} \notin\{v\}^{\perp} .
$$

In fact $\bar{K}$ is not orthogonal to $v$ because $\operatorname{hom}(1, \bar{K})=\emptyset$. And $\bar{K}$ is orthogonal to $h_{i}$ because the unique morphism from $\left(A_{i}, \alpha_{i}, a_{i}\right)$ is that obtained from id $A_{i}$ in $\mathcal{K}$. Indeed, let $f:\left(A_{i}, \alpha_{i}, a_{i}\right) \rightarrow \coprod_{j \in J}\left(A_{j}, \alpha_{j}, a_{j}\right)$ be a morphism of type (d). Since $a_{i}$ is a clique on $A_{i}$, it easily follows that there exists $j \in J$ with $f\left[A_{i}\right] \subseteq A_{j}$. This implies $J=\{j\}$ by our minimality requirement above. Since $\left(A_{i}, a_{i}\right)$ is a clique and $\left(A_{j}, a_{j}\right)$ has no loops, $f$ must be one-to-one. This implies $i=j$ and $f=\mathrm{id}_{A_{i}}$ by our choice at the beginning.

5.9. Theorem In locally presentable categories the Orthogonality Logic is complete for all quasi-presentable classes of morphisms.

Proof In [2] we proved the completeness for presentable classes. To generalize this to quasi-presentable classes, we use the idea (and notation) of the proof of Theorem 5.3, part (2). Given

$$
\mathcal{H} \models k,
$$

since $\mathcal{H}_{e}^{\perp}=\hat{\mathcal{H}}_{e}^{\perp}$, we have that $\mathcal{H}_{0} \cup \hat{\mathcal{H}}_{e} \models k$. Hence, by the completeness for presentable classes, $\mathcal{H}_{0} \cup \hat{\mathcal{H}}_{e} \vdash k$. To conclude that $\mathcal{H} \vdash k$, it suffices to show that $\mathcal{H}_{e} \vdash g$ for each member $g$ of $\hat{\mathcal{H}}_{e}$. Now $g \in \hat{\mathcal{H}}_{e}$ implies there exists a colimit cocone $c_{h}:\left(A \stackrel{h}{\longrightarrow} C_{h}\right) \longrightarrow(A \stackrel{f}{\longrightarrow} B)$ in $A \downarrow \mathcal{A}$ with $f \in \mathcal{H}_{e}$ such that $g$ is one of the morphisms $h$. Consequently, $f=c_{g} \cdot g$. Since $g$ is an epimorphism, from $\mathcal{H} \vdash f$ we deduce $\mathcal{H} \vdash g$, see [2], 3.6(vii). 


\section{A Counter-example}

A generalization of the Small Object Argument was presented in [4] for sets of morphisms in categories "almost like" locally bounded ones: they are called locally ranked and the definition differs from 2.2 above only in modifying (ii) as follows:

for every object $A$ there is a regular cardinal $\lambda$ (called rank) such

that $\operatorname{hom}(A,-)$ preserves $\lambda$-directed colimits of monomorphisms

in $\mathcal{M}$.

We are going to demonstrate that, in the spite of the formal similarity, Theorems 3.6 and 5.1 fail in general for locally ranked categories. We are using unary algebras defined on cpo's: the corresponding category UCPO will be proved to have the form Inj $\mathcal{H}$ for a quasi-small class $\mathcal{H}$ in a locally ranked category, and yet, UCPO is not weakly reflective there.

In the following definition we assume the standing hypotheses formulated in Section 2:

6.1. Definition $([4])$. We call $\mathcal{A}$ locally $\mathcal{E}$-ranked provided that every object in $\mathcal{A}$ has a rank. Locally ranked means locally Epi-ranked.

6.2. Example We give an example of a locally ranked category

\section{$\mathrm{UCPO}^{*}$}

and its quasi-small class $\mathcal{H}$ of morphisms with

$$
\mathrm{UCPO}=\mathcal{H}^{\perp}
$$

such that UCPO is not reflective in UCPO*.

(a) We recall the well-known category CPO whose objects, the (strict) cpo's, are posets with a least element $\perp$ and with joins of directed subsets. Morphisms are functions which are

(i) continuous, i.e., preserve directed joins, and

(ii) strict, i.e., preserve $\perp$.

We denote by

\section{UCPO}

the category of unary algebras on cpo's: objects are pairs $(A, \alpha)$ where $A$ is a cpo and $\alpha$ is an order-preserving unary operation on $A$. Morphisms are the continuous and strict algebra homomorphisms. 
(b) Our next category is the category

\section{$\mathrm{CPO}^{*}$}

of partial cpo's. These are pairs $\left(A, Z_{A}\right)$ consisting of a poset $A$ with a least element $\perp$, and a set $Z_{A} \subseteq \exp A$ of directed subsets which have a join in $A$. Every сро is considered as a partial cpo with $Z_{A}=$ all directed subsets of $A$.

Morphisms $f: A \rightarrow B$ of $\mathbf{C P O}^{*}$ are the strict, order-preserving functions which are continuous in the expected sense: if $M \in Z_{A}$, then $f[M] \in Z_{B}$ and $f$ preserves the join of $M$.

(c) Our main category is the category

\section{$\mathrm{UCPO}^{*}$}

of all pairs $(A, \alpha)$ where $A$ is a partial cpo and $\alpha$ is a binary relation on its underlying set. Every unary cpo-algebra is considered as an object with $\alpha$ equal to the graph of the unary operation.

Morphisms $f:(A, \alpha) \rightarrow(B, \beta)$ are the strict, continuous functions $f$ : $A \rightarrow B$ which are graph homomorphisms, that is, from $a \alpha a^{\prime}$ it follows that $f(a) \beta f\left(a^{\prime}\right)$.

6.3. Notation (1) For every partial cpo, $A$, we denote by

$$
\varrho_{A}: A \rightarrow \bar{A}
$$

its reflection in $\mathrm{CPO}$. That is:

(i) $\bar{A}$ is a cpo,

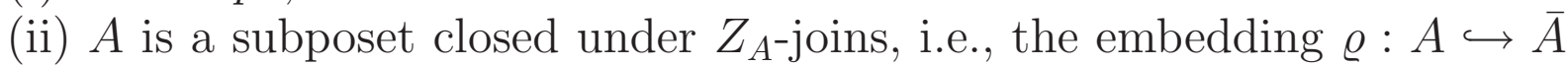
is strict and continuous, and

(iii) every strict continuous function $f: A \rightarrow B$ where $B$ is a cpo has a unique strict continuous extension to $\bar{f}: \bar{A} \rightarrow B$.

This poset $\bar{A}$ always exists, see [18]. In the articles [18] and [19] Ana Pasztor works with a subset system $Z$ on the category of posets, but all her results remain true if one works with one poset $A$ and one collection $Z_{A}$.

(2) Let $\mathcal{H}_{e}$ be the class of all morphisms

$$
\varrho_{A}:(A, \emptyset) \rightarrow(\bar{A}, \emptyset)
$$

where $A$ is a partial cpo considered as an object of $\mathbf{U C P O}^{*}$ via the empty relation. 
(3) Let $h: P \rightarrow Q$ denote the following morphism in $\mathbf{U C P O}^{*}$ carried by the inclusion map

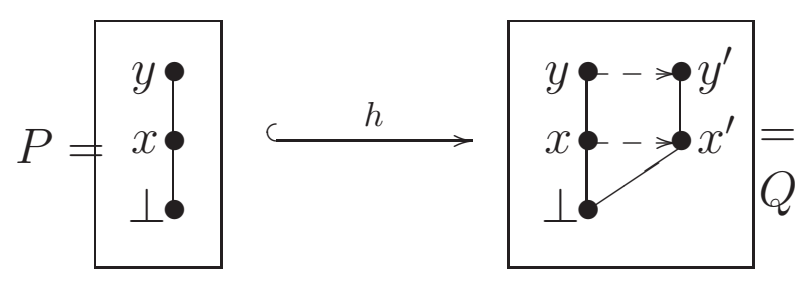

where the edges $\mid$ and $-->$ correspond to the ordering $\leq$ and the relation $\alpha$, respectively.

6.4. Lemma We have $\mathbf{U C P O}=\mathcal{H}^{\perp}$ for $\mathcal{H}=\mathcal{H}_{e} \cup\{h\}$ and every member of $\mathcal{H}_{e}$ is an epimorphism in $\mathbf{U C P O}^{*}$. However $\mathbf{U C P O}$ is not reflective in UCPO*.

Remark In fact, we will see that UCPO is not weakly reflective in UCPO*. Proof An object $(A, \alpha)$ is orthogonal to $\mathcal{H}_{e}$ iff $A$ is a $c p o$ : this follows from the fact that $\mathcal{H}_{e}$ is the class of reflections of all objects of $\mathbf{C P O}^{*}$ in the subcategory CPO. Let $A$ be a cpo and let

$$
(A, \alpha) \perp h \text {. }
$$

For every $a \in A$ the morphism $P \rightarrow A$ with $x \mapsto a$ and $y \mapsto a$ factors uniquely through $h$, thus, there exists a unique $a^{\prime}$ with $a \alpha a^{\prime}$. This tells us that $\alpha$ is (the graph of) a unary operation. And $\alpha$ is obviously order preserving: for $a \leq b$ in $A$ consider the morphism $P \rightarrow A$ with $x \mapsto a, y \mapsto b$. Conversely, whenever $\alpha$ is an order preserving unary operation, then clearly $(A, \alpha) \perp h$. This proves

$$
\mathcal{H}^{\perp}=\mathrm{UCPO} .
$$

To see that this is not a reflective subcategory of the category $\mathbf{U C P O}^{*}$, observe that the latter category has a (one-element) initial object, and the category UCPO does not have any weakly initial object. In fact, suppose $(A, \alpha)$ is initial in UCPO and define a chain of elements $a_{i} \in A$ for all ordinals $i$ by transfinite induction as follows:

$$
\begin{aligned}
& a_{0}=\perp, \\
& a_{i+1}=\alpha a_{i},
\end{aligned}
$$

and for limit ordinals $j$ 


$$
a_{j}=\bigvee_{i<j} a_{i}
$$

It follows by easy transfinite induction that $i \leq i^{\prime}$ implies $a_{i} \leq a_{i^{\prime}}$. Thus, the last join exists. We prove that $i<j$ implies $a_{i} \neq a_{j}-$ a contradiction. In fact, let $(B, \beta)$ be the object of $\mathbf{U C P O}$ where $B$ is the cpo of all ordinals $k \leq j$ and $\beta$ is defined by $\beta(k)=k+1$ for $k<j$ and $\beta(j)=j$. The unique morphism $f:(A, \alpha) \rightarrow(B, \beta)$ fulfils $f\left(a_{k}\right)=k$ for all $k \leq j$, as easily proved by transfinite induction on $k$. Since $f\left(a_{i}\right)<f\left(a_{j}\right)$, we conclude $a_{i} \neq a_{j}$.

Finally, each $\varrho_{A} \in \mathcal{H}_{e}$ is an epimorphism because given morphisms $u, v$ : $(\bar{A}, \emptyset) \rightarrow(B, \beta)$ with $u \cdot \varrho_{A}=v \cdot \varrho_{A}$, the morphisms $\varrho_{B} \cdot u, \varrho_{B} \cdot v: \bar{A} \rightarrow \bar{B}$ are continuous, therefore $\left(\varrho_{B} \cdot u\right) \cdot \varrho_{A}=\left(\varrho_{B} \cdot v\right) \cdot \varrho_{A}$ implies $\varrho_{B} \cdot u=\varrho_{B} \cdot v$ by the universal property of $\varrho_{A}$. Since $\varrho_{B}$ is a monomorphism, we conclude $u=v$.

6.5. Theorem The category $\mathrm{UCPO}^{*}$ is locally ranked, and $\mathcal{H}=\mathcal{H}_{e} \cup\{h\}$ is a class of morphisms whose members are all but one epimorphisms and such that $\mathcal{H}^{\perp}$ is not reflective.

Proof In view of the above lemma, it remains to show that $\mathbf{U C P O}^{*}$ is locally ranked.

(1) Proof of the cocompleteness of CPO*: coproducts in $\mathbf{C P O}^{*}$ are the obvious disjoint unions with all bottom elements merged to one. To describe coequalizers, let $u, v: A \rightarrow B$ be strict and continuous functions and let $\bar{u}, \bar{v}: \bar{A} \rightarrow \bar{B}$ be their unique strict and continuous extensions, see 6.3. Form a coequalizer, $\bar{c}: \bar{B} \rightarrow \bar{C}$, of $\bar{u}$ and $\bar{v}$ in the (cocomplete) category CPO:

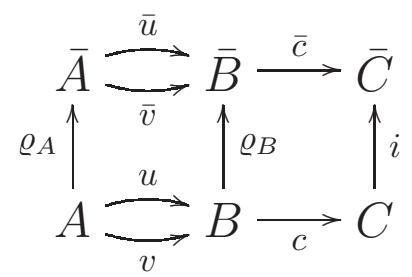

Denote by $i: C \hookrightarrow \bar{C}$ the subposet of $\bar{C}$ on the image of $\bar{c} \cdot \varrho_{B}$, considered as a partial cpo via

$$
Z_{C}=\left\{c[M] ; M \in Z_{B}\right\} .
$$

Then $c: B \rightarrow C$ is a morphism of $\mathbf{C P O}^{*}$ : it is strict and order-preserving (being equal to $\bar{c} \cdot \varrho_{B}$ ). Also, the definition of $Z_{C}$ makes $c$ obviously continuous (since $\bar{c}$ is). We claim that $c$ is a coequalizer of $u$ and $v$. 
Given a morphism $d: B \rightarrow D$ of $\mathbf{C P O}^{*}$ with $d \cdot u=d \cdot v$, the corresponding morphism $\bar{d}: \bar{B} \rightarrow \bar{D}$ fulfils $\bar{d} \cdot \bar{u}=\bar{d} \cdot \bar{v}$ :

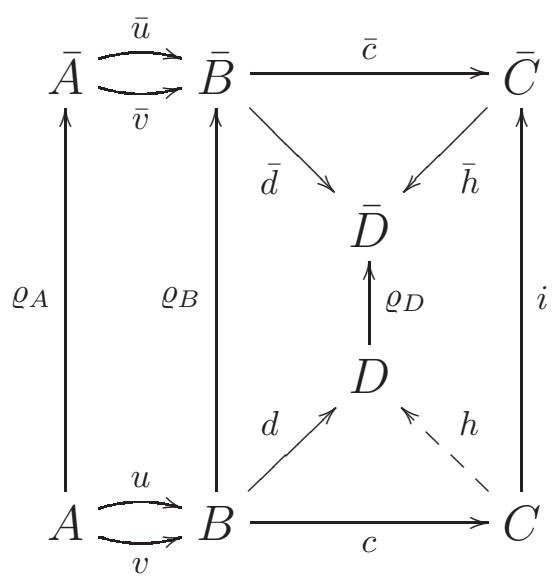

Therefore, there is a strict, continuous $\bar{h}: \bar{C} \rightarrow \bar{D}$ with $\bar{h} \cdot \bar{c}=\bar{d}$. Since $c$ is surjective and $r_{D}$ monic, there exists a diagonal fill-in morphism $h$ in Set, and we only need to show that $h$ is strict (which is obvious from the strictness of $d$ ) and continuous. In fact, for every member of $Z_{C}$ of the form $c[M], M \in Z_{B}$, we know that $d[M] \in Z_{D}$ and $d(\vee M)=\vee d[M]$. From $d=h \cdot c$ we conclude

$$
(h \cdot c)[M] \in Z_{D}
$$

and

$$
\vee(h \cdot c)[M]=\vee d[M]=d(\vee M)=h \cdot c(\vee M) .
$$

Finally, $\bar{c} \cdot \varrho_{B}$ is strict and continuous, therefore $\bar{c} \cdot \varrho_{B}$ preserves the join of $M$. Consequently, $\vee M$ lies in the image of $B$ under $\bar{c} \cdot \varrho_{B}$, which is $C$. We conclude that $c$, the codomain restriction of $\bar{c} \cdot \varrho_{B}$, also preserves the join of $M$, finishing the proof of

$$
h \cdot c(\vee M)=h(\vee c[M]) .
$$

(2) $\mathbf{U C P O}^{*}$ is cocomplete. In fact, the forgetful functor to $\mathbf{C P O}^{*}$ makes

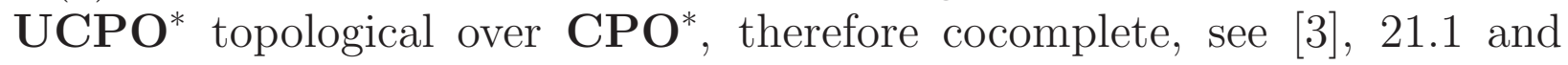
21.16 .

(3) $\mathrm{UCPO}^{*}$ is cowellpowered. It is sufficient to prove that $\mathbf{C P O}^{*}$ is cowellpowered, see [3], 21.16.

We will use the description of epimorphisms of $\mathbf{C P O}^{*}$ presented by Ana Pasztor [19]. Let

$$
f: A \rightarrow B \quad \text { be a morphism in } \mathrm{CPO}^{*}
$$


and for every set $Y \subseteq B$ let

$$
\operatorname{cl}(Y)
$$

be the least subset $W$ of $B$ containing $Y$ such that $\vee M \in W$ for every set $M \in Z_{B}$ contained in $Y$. The morphism $f$ is an epimorphism iff

$$
\text { for every } b \in B \text { there exists an ordinal } i \text { with } b \stackrel{i}{\longrightarrow} b \text {, }
$$

where $\stackrel{i}{\longrightarrow}$ are relations on the underlying set of $B$ defined by transfinite induction as follows:

(i) Intial case: $b \stackrel{0}{\longrightarrow} b^{\prime}$ iff $b \geq f(a) \geq b^{\prime}$ for some $a \in A$.

(ii) Successor ordinals: $b \stackrel{i+1}{\longrightarrow} b^{\prime}$ iff $b^{\prime} \in \operatorname{cl}(Y)$ where $Y=\{y \in B ; \quad b \stackrel{i}{\longrightarrow} y\}$

(iii) Limit ordinals $j: \stackrel{j}{\longrightarrow}$ is the union of all $\stackrel{i}{\longrightarrow}$ with $i<j$.

Let $\hat{A}$ be a free completion of $A$ under joins and let $\hat{f}: \hat{A} \rightarrow \hat{B}$ be the extension of $f$ preserving joins. We prove that every element of $B$ lies in the image of $\hat{f}$ by transfinite induction on $i$ with $b \stackrel{i}{\longrightarrow} b$. This follows from the following more general fact:

$$
b \stackrel{i}{\longrightarrow} b^{\prime} \quad \text { implies } \quad b \geq \hat{f}(\hat{a}) \geq b^{\prime} \quad \text { for some } \quad \hat{a} \in \hat{A} \text {. }
$$

Case $i=0$ is obvious.

Case $i+1$ : Given $Y \subseteq B$ with $b \stackrel{i}{\longrightarrow} y$ for $y \in Y$, there exist $\hat{a}_{y} \in \hat{A}$ with $b \geq \hat{f}\left(\hat{a}_{y}\right) \geq y$, by induction hypothesis. Let $\hat{a}=\bigvee_{y \in Y} \hat{a}_{y}$. We have $b \geq \hat{f}(\hat{a})$, since $\hat{f}$ preserves joins, and we prove $\hat{f}(\hat{a}) \geq b^{\prime}$. In fact, the set $C$ of all $c \in B$ with $\hat{f}(\hat{a}) \geq c$ contains $Y$ and is closed under joins, therefore, it contains $\operatorname{cl}(Y)$. Thus, we just use $b^{\prime} \in \operatorname{cl}(Y)$.

The limit case is obvious.

Since card $\hat{A} \leq 2^{\text {card } A}$, we have just established that for every epimorphism $f: A \rightarrow B$ we have $\operatorname{card} B \leq 2^{\text {card } A}$. This proves the cowellpoweredness of $\mathrm{CPO}^{*}$.

(4) Strong subobjects of UCPO*: Suppose that

$$
m:(C, \gamma) \rightarrow(D, \delta)
$$

is a strong monomorphism. The diagonal fill-in property w.r.t. the epimorphism 


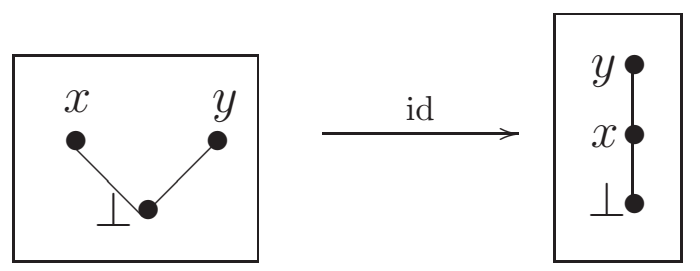

tells us that for every $x, y \in C$ we have

$$
x \leq y \text { in } C \text { iff } m(x) \leq m(y) \text { in } D .
$$

Consequently, we can assume without loss of generality that $C$ is a subposet of $D$ and $m$ is the inclusion map. Moreover, $C$ is closed under the chosen joins in the following sense: put

$$
Z_{C}=Z_{D} \cap(\exp C)
$$

then the join of a set $M \in Z_{C}$ is the same in $C$ and $D$. In fact, this follows from the diagonal fill-in property applied to the epimorphism

$$
M \cup\{\perp\} \hookrightarrow M \cup\{\perp, m\}
$$

where the domain, $A$, is the subposet of $D$ with $Z_{A}=\emptyset$, and the codomain, $B$, is the subposet obtained from $A$ by adding $m=\vee M$ and putting $Z_{B}=\{M\}$. Finally, the relation $\gamma$ is simply $\delta \cap(C \times C)$ : use the epimorphism

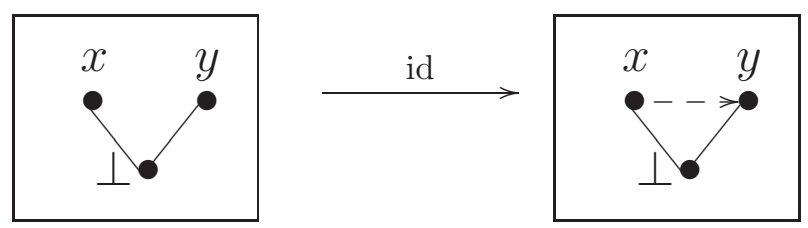

(5) Colimits of directed diagrams $D$ of strong subobjects are unions. We can suppose that $D$ has objects $\left(D_{i}, \delta_{i}\right)$ where $i$ ranges through a $\lambda$-directed poset $I$ (with $\lambda$ an infinite cardinal), and that for $i \leq j$ the connecting map $\left(D_{i}, \delta_{i}\right) \rightarrow\left(D_{j}, \delta_{j}\right)$ is an embedding of a subposet $D_{i}$ of $D_{j}$ closed under $Z_{D_{j}}$-joins and with the induced set $Z_{D_{i}}$, see (4) above. Let

$$
C=\cup_{i \in I} D_{i}
$$

be the poset with the ordering and $Z_{C}$ induced by the $D_{i}$ 's:

$$
x \leq y \text { in } C \quad \text { iff } \quad x \leq y \text { in } D_{i} \text { for some } i
$$

and

$$
Z_{C}=\bigcup_{i \in I} Z_{D_{i}}
$$


For every $M \in Z_{C}$ we have a join $m$ of $M$ in some $D_{i}$, and the same $m$ is the join of $M$ in $D_{j}$ for all $j \geq i$ - therefore $m=\vee M$ in $C$. We now define the binary relation $\gamma$ on $C$ as that induced by the given binary relations: $\gamma=\cup_{i \in I} \delta_{i}$. It is easy to verify that the embbeddings

$$
\left(D_{i}, \delta_{i}\right) \hookrightarrow(C, \gamma)
$$

form a colimit in $\mathrm{UCPO}^{*}$.

(6) Every object $(A, \alpha)$ of cardinality

$$
\operatorname{card} A<\lambda \quad(\lambda \text { an infinite cardinal })
$$

has rank $\lambda$. This follows immediately from (5): given a morphism $f$ : $(A, \alpha) \rightarrow(C, \gamma)$, the image of $f$ lies in $D_{i}$ for some $i \in I$. Then the fact that the subposet $D_{i}$ is closed in $C$ as well as in each $D_{j}, j \geq i$, under the directed sets of $Z_{D_{i}}$, their joins, and the relation (as proved in (4)) makes it clear that

$$
f^{\prime}:(A, \alpha) \rightarrow\left(D_{i}, \delta_{i}\right)
$$

is a morphism. Therefore, $\operatorname{hom}(A,-)$ preserves the colimit as described in $(5)$.

6.6. Remark We now want to have an analogous example for injectivity: a quasi-small class $\mathcal{H}$ with $\operatorname{Inj} \mathcal{H}=\mathbf{U C P O}$. In fact, all we need is to add to the above class $\mathcal{H}_{e}$ an epimorphism $f$ such that injectivity w.r.t. $f$ is equivalent to the statement that the binary relation is (the graph of a) unary operation. Here is such an epimorphism:

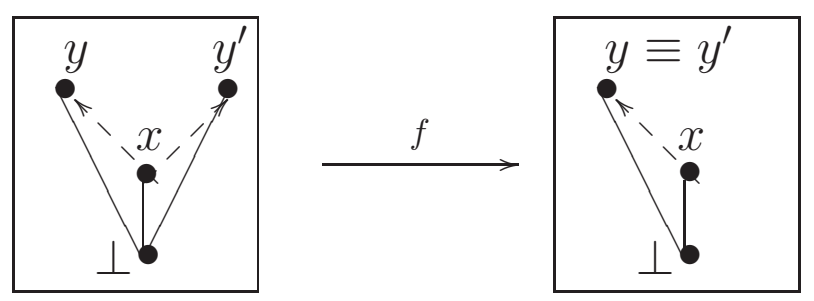

6.7. Corollary In the locally ranked category UCPO* $^{*}$ the class $\mathcal{H}=\mathcal{H}_{e} \cup$ $\{f, h\}$ has all members except $h$ epimorphisms; however $\mathbf{U C P O}=$ Inj $\mathcal{H}$ is not weakly reflective.

In fact, it follows by using the argument in the proof of Lemma 6.4. 


\section{Summary and Open Problems}

An excellent solution of the orthogonal subcategory problem was presented by Max Kelly and Peter Freyd [9], [15]: for every quasi-small class $\mathcal{H}$ of morphisms in a locally bounded category the orthogonal subcategory of $\mathcal{H}$ is reflective. Based on the constructive approach to reflections due to Max Kelly we have proved that, under the same assumptions, our Orthogonality Logic [2] is complete. It appears that local boundedness is a more suitable concept than that of a locally ranked category, introduced in [4]: we have presented an example of a quasi-small class $\mathcal{H}$ in the locally ranked category UCPO* $^{*}$ whose orthogonal subcategory is not reflective.

In locally presentable categories of $\mathrm{P}$. Gabriel and F. Ulmer a stronger result holds: whenever $\mathcal{H}$ is a quasi-presentable class, then its orthogonal subcategory is reflective and the Orthogonality Logic is complete. Moreover, under the set-theoretical Vopěnka's Principle no restrictions on the class $\mathcal{H}$ of morphisms are needed: (i) the orthogonal subcategory is always reflective, and (ii) the Orthogonality Logic is always complete. Actually, the statement (i) is equivalent to Weak Vopěnka's Principle, see 6.22-23 in [5], and the statement (ii) is equivalent to Vopěnka's Principle, see 4.6 in [2].

Open Problem Under which conditions on the base category does the reflectivity of $\mathcal{H}^{\perp}$ always imply the completeness of the Orthogonality Logic for $\mathcal{H}$ ? Under which conditions does the converse implication always hold?

The main result of our paper is that Quillen's Small Object Argument can be generalized to precisely the same situations studied by Freyd and Kelly: in every locally bounded category, given a quasi-small class $\mathcal{H}$ of morphisms, then cellular weak $\mathcal{H}$-injective reflections exist, and the Injectivity Logic is complete. Again, we have proved a stronger result for locally presentable categories: here quasi-presentability of the class of morphisms is sufficient.

As mentioned above, in [4] the authors gave, for every set $\mathcal{H}$ of morphisms in a locally ranked category, a construction for a cellular weak reflection in the subcategory Inj $\mathcal{H}$. But they gave as well a construction for a functorial (they called it "natural") weak reflection, and stated as an open problem whether there exists a weak reflection which would be simultaneously cellular and functorial. It appears that we have a similar situation here: given a quasi-presentable class $\mathcal{H}$ of morphisms in a locally presentable category, 
Theorem 3.11 gives a construction for a cellular weak reflection into Inj $\mathcal{H}$, and Theorem 4.4 a functorial one. However, we do not know if there exists a cellular and functorial weak reflection.

The situations for injectivity and for orthogonality are no longer symmetric when arbitrary classes of morphisms are considered. We now have that: (i) Vopěnka's Principle implies that the injectivity subcategory is always weakly reflective, which itself implies Weak Vopěnka's Principle (see 6.26-27 in [5]); however, (ii) the Injectivity Logic is not always complete (independently of set-theory): Examples 3.8 and 4.5 describe a (weakly reflective) subcategory of the locally finitely presentable category of graphs of the form Inj $\mathcal{H}$ for which the Injectivity Logic is not complete. Thus here we are left with the following:

Open Problem Under which conditions on the base category does the completeness of the Injectivity Logic always imply the weak reflectivity of the injective subcategory?

\section{References}

[1] J. Adámek, M. Hébert and L. Sousa, A Logic of Injectivity, accepted for publication in Journal of Homotopy and Related Structures.

[2] J. Adámek, M. Hébert and L. Sousa, A Logic of Orthogonality, Archivum Mathematicum, Tomus 42 (2006), 309-334.

[3] J. Adámek, H. Herrlich and G. E. Strecker, Abstract and Concrete Categories, John Wiley and Sons, New York 1990. Freely available at www.math.uni-bremen.de/ dmb/acc.pdf

[4] J. Adámek, H. Herrlich, J. Rosický, W. Tholen, On a Generalized Small-Object Argument for the Injective Subcategory Problem, Cah. Topol. Géom. Diffèr. Catég. 43 (2002), 83-106.

[5] J. Adámek and J. Rosický: Locally presentable and accessible categories, Cambridge University Press, 1994.

[6] J. Adámek, J. Rosický and V. Trnková, Topological reflections revisited, Trans. Amer. Math. Soc. 108 (1990), 605-612.

[7] J. Adámek and L. Sousa, On reflective subcategories of varieties, J. Algebra 276 (2004) 685705.

[8] J. Adámek, M. Sobral and L. Sousa, Logic of implications, Preprints of the Department of Mathematics of the University of Coimbra 05-24 (2005).

[9] P. J. Freyd and G.M. Kelly, Categories of continuous functors I, J. Pure Appl. Algebra 2 (1972), 169-191.

[10] P. Gabriel and F. Ulmer, Local Präsentierbare Kategorien, Lect. Notes in Math. 221, SpringerVerlag, Berlin 1971.

[11] P. Gabriel and M. Zisman, Calculus of Fractions and Homotopy Theory, Springer Verlag 1967.

[12] M. Hébert, Algebraically closed and existentially closed substructures in categorical context, Theory Appl. Cat. 12 (2004), 270-298.

[13] M. Hébert, $\mathcal{K}$-Purity and orthogonality, Theory Appl. Cat. 12 (2004), 355-371. 
[14] M. Hébert, $\lambda$-presentable morphisms, injectivity and (weak) factorization systems, Appl. Cat. Struct. 14 (2006), 273-289.

[15] M. Kelly, A unified treatment of transfinite constructions for free algebras, free monoids, colimits, associated sheaves, and so on, Bull. Austral. Math. Soc. 22 (1980), 1-84.

[16] V. Koubek, Every concrete category has a representation by $T_{2}$ paracompact topological spaces, Comment. Math. Univ. Carolinae 15 (1975), 655-664.

[17] V. Koubek, J. Reiterman, Categorical constructions of free algebras, colimits, and completions of partial algebras, J. Pure Appl. Algebra 14 (1979), 195-231.

[18] A. Pasztor, The epis of the categories of ordered algebras and Z-continuous homomorphisms, Cahiers Topol. Geom. Different. Categ. 24 (1983), 203-214.

[19] A. Pasztor, Epis of some categories of $Z$-continuous partial algebras, Acta Cybernetica 6 (1983), 111-123.

[20] D. Quillen, Homotopical algebra, Lecture Notes Math. 43, Springer-Verlag, Berlin 1967.

[21] G. Roşu, Complete Categorical Equational Deduction, Lecture Notes in Comput. Sci. 2142 (2001), 528-538.

[22] L. Sousa, Solid hulls of concrete categories, Appl. Categ. Structures 3 (1995), 105-118.

JIŘÍ ADÁMEK

Department of Theoretical Computer Science, Technical University of Braunschweig, Postfach 3329, 38023 Braunschweig, Germany

MiChEL HÉBERT

Mathematics Department, The American University in Cairo, P.O.Box 2511, Cairo 11511 , EGYPT

Lurdes Sousa

Departamento de Matemática, Escola Superior de Tecnologia de Viseu, Campus Politécnico, 3504-510 Viseu, PorTugal

CMUC, University of Coimbra, 3001-454 Coimbra, Portugal 